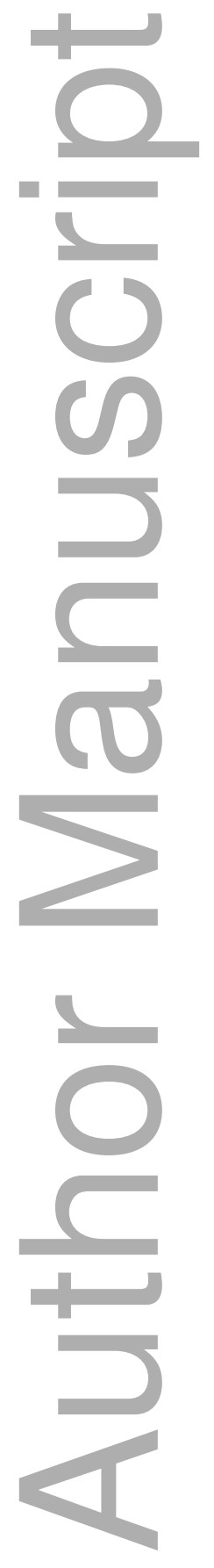

This is the author manuscript accepted for publication and has undergone full peer review but has not been through the copyediting, typesetting, pagination and proofreading process, which may lead to differences between this version and the Version of Record. Please cite this article as doi: $\underline{10.1002 / M P .13413}$

This article is protected by copyright. All rights reserved 


\title{
Cherenkov emission-based external radiotherapy dosimetry: II. Electron beam quality specification and uncertainties
}

\author{
Yana Zlateva \\ Medical Physics Unit, McGill University, Montreal, QC, H4A 3J1, Canada, ${ }^{*}$ \\ Metrology Research Centre, Nationa \\ Bryan R. Muir \\ Jan P. Seuntjens \\ Issam El Naqa
}

5

( a companion study (Paper I). each detection configuration.

Department of Radiation Oncology, University of Michigan, Ann Arbor, MI, 48103-4943, USA

(Dated: January 28, 2019)

Purpose: Cherenkov emission (CE) is ubiquitous in external radiotherapy. It is also unique in that it carries the promise of 3D micrometer-resolution perturbation-free in-water dosimetry with a beam quality-independent detector response calibration. Our aim is to bring CE-based dosimetry into the clinic and we motivate this here with electron beams. We Monte Carlo-calculate and characterize broad-beam CE-to-dose conversion factors in water for a clinically representative library of electron beam qualities, address beam quality specification and reference depth selection, and develop a preliminary uncertainty budget based on our Monte Carlo results and relative experimental work of

Methods: Broad electron beam CE-to-dose conversion factors $k_{\mathrm{C}}^{\theta \pm \delta \theta}$ include CE generated at polar angles $\theta \pm \delta \theta$ on beam axis in water. With modifications to the EGSnrc code SPRRZnrc, $k_{\mathrm{C}}^{\theta \pm \delta \theta}$ factors are calculated for a total of 20 electron beam qualities from 4 BEAMnrc models (Varian Clinac 2100C/D, Clinac 21EX, TrueBeam, and Elekta Precise). We examine beam quality, depth, and detection angle dependence for $\theta \pm \delta \theta=90^{\circ} \pm 90^{\circ}$ ( $4 \pi$ detection), $90^{\circ} \pm 5^{\circ}, 45^{\circ} \pm 45^{\circ}$, and $90^{\circ} \pm 45^{\circ}$. As discussed in Paper I, $4 \pi$ detection offers the strongest CE-dose correlation and $\theta=90^{\circ}$ with small $\delta \theta$ is most practical. The two additional configurations are considered as a compromise between these two extremes. We address beam quality specification and reference depth selection in terms of the electron beam quality specifier $R_{50}$, obtained from the depth of $50 \% \mathrm{CE} C_{50}$, and derive a best-case uncertainty budget for the CE-based dosimetry formalism proposed in Paper I at

Results: The $k_{\mathrm{C}}^{\theta \pm \delta \theta}$ factor was demonstrated to capture variations in the beam spectrum, angle, photon contamination, and electron fluence below the $\mathrm{CE}$ threshold ( $\sim 260 \mathrm{keV}$ in the visible) in accordance with theory. The root-mean-square deviation and maximum deviation of a second-order polynomial fit of simulated $R_{50}$ values in terms of $C_{50}$ were $0.05 \mathrm{~mm}$ and $0.11 \mathrm{~mm}$ at $4 \pi$ and $0.20 \mathrm{~mm}$ and $0.33 \mathrm{~mm}$ at $90^{\circ} \pm 5^{\circ}$ detection, respectively. The fit performance on experimental data in Paper I was in agreement with these values within experimental uncertainties $( \pm 1.5 \mathrm{~mm}, 95 \% \mathrm{CI})$. A twoterm power function fit of $k_{\mathrm{C}}^{\theta \pm \delta \theta}$ in terms of $R_{50}$ at a reference depth $d_{\text {ref }}=a R_{50}+b$ resulted in total

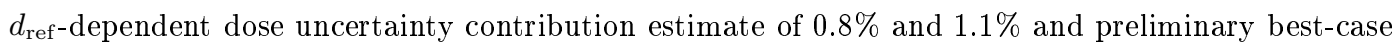
estimate of the combined standard dose uncertainty of $1.1 \%$ and $1.3 \%$ at $4 \pi$ and $90^{\circ} \pm 5^{\circ}$ detection, respectively. The results and corresponding uncertainties with the two intermediate apertures were

\section{This article is protected by copyright. All rights reserved}


generally of the same order as the $4 \pi$ case. In addition, a theoretically consistent downstream shift of the PDC by the difference between $R_{50}$ and $C_{50}$ improved the depth dependence of the $4 \pi$ conversion by an order of magnitude $( \pm 2.8 \%)$. Therefore, a large aperture centered on a $\theta$ value between $45^{\circ}$ and $90^{\circ}$ combined with a downstream PDC shift may be recommended for beam-axis CE-based electron beam dosimetry in water.

Conclusions: By delivering $R_{50}$-based CE-to-dose conversion data and demonstrating the potential for dosimetric uncertainty on the order of $1 \%$, we bring CE-based electron beam dosimetry closer to clinical realization.

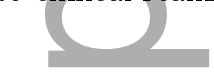

$\square$
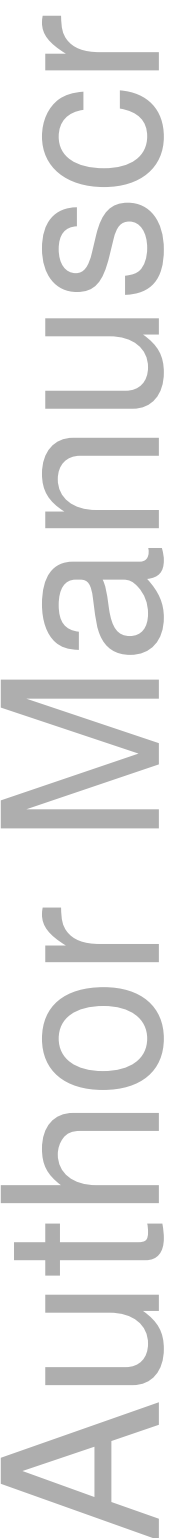

* yana.zlateva@mail.mcgill.ca; http://www.mcgill.ca/medphys/ 


\section{INTRODUCTION}

The raw reading, $M_{\text {raw }}$, is background-subtracted and corrected to the reference conditions of $S S D$, point of measurement, field size, $F S$, and temperature, $T$, for which the calibration, $N$, and the CE-to-dose conversion, $k_{\mathrm{C}}^{\theta \pm \delta \theta}$, apply. The $k_{\mathrm{C}}^{\theta \pm \delta \theta}$ factor includes only and all CE generated at polar angles in the range $\theta \pm \delta \theta$ relative to the beam direction. Azimuthal integration is implied or alternatively the azimuthal component of the detection optics angular aperture (i.e., acceptance angle) can be included in the calibration, $N$. The setup and involved quantities are described in 
detail in Paper I with angles defined in Figure 2 of Paper I.[6] The temperature correction factor, $P_{\mathrm{T}}$, is discussed in are not relevant in CE-based dosimetry in water.

Motivated by the experimental validation of Paper I,[6] in this work we conduct Monte Carlo calculations and characterize the $k_{\mathrm{C}}$ factor for a library of validated electron beam models representative of clinical beams and we investigate the optimal detection configuration. We then address beam quality specification and evaluate an optimal reference depth, where the CE-based dose measurement uncertainty is minimized for electron beams, in terms of the beam quality specifier $R_{50}$. Finally, based on our Monte Carlo and relative experimental results, we estimate a potential uncertainty budget for the proposed CE-based dosimetry formalism.

\section{MATERIALS AND METHODS}

The envisioned setup for the purpose of CE-based dosimetry at $\theta \pm \delta \theta$ detection on beam axis in water, using optical sectioning or tomographic techniques, as well as the experimental validation setup and performance are discussed in Paper I.[6]

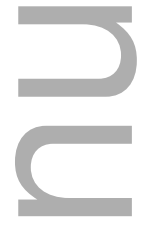

\section{A. BEAMnrc models and parameters}

A clinically representative library of electron beams was simulated with the BEAMnrc code[13, 14] of the particle transport simulation package EGSnrc.[15, 16] BEAMnrc models include a Varian Clinac 2100C/D at Karolinska University Hospital in Sweden (downloaded from the IAEA phase-space database),[17] Varian Clinac 21EX at the Montreal General Hospital,[18] Varian TrueBeam data provided by the vendor (Varian Medical Systems, Inc., Palo Alto, CA),[19] and Elekta Precise at the National Research Council of Canada.[20] Twenty electron beams were simulated with nominal energies of $4-20 \mathrm{MeV}$ and $22 \mathrm{MeV}$ at $10 \times 10-\mathrm{cm}^{2}$ and $20 \times 20-\mathrm{cm}^{2}$ field size, respectively, and at SSD of $100 \mathrm{~cm} \cdot[21]$ This list of models, summarized in Table I, accounts for variations in machine types and manufacturers, encompassing most of the relevant clinical electron beams. The value of the depth of $50 \%$ dose, $R_{50}$, in Table I was estimated from a linear interpolation of the simulated percent-depth dose (PDD) in water.

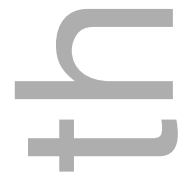

\section{B. SPRRZnrc modifications and parameters}

CE-to-dose conversion factors $k_{\mathrm{C}}^{\theta \pm \delta \theta}$ are calculated in water with a modified version[22] of the SPRRZnrc code,[23] adapted for this purpose as described in Paper I.[6] The Spencer-Attix cut-off $\Delta$ for scoring the dose[24] was set to $10 \mathrm{keV}$ [6] and the CE threshold for scoring CE was set to $257 \mathrm{keV}$ by using a refractive index value of $n=1.34$,[2] which corresponds to a minimum, at $475 \mathrm{~nm}$, in optical absorption by water at room temperature.[25, 26]

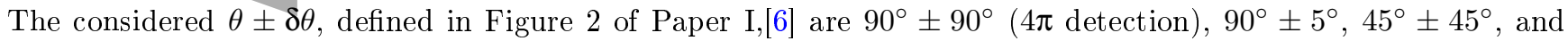
$90^{\circ} \pm 45^{\circ}$. As discussed in Paper I, a $4 \pi$ configuration requires $4 \pi$ detection or additional uncertainty considerations. On the other hand, $42^{\circ}$ is the CE angle of relativistic electrons in water, [3, 25] resulting in the highest signal within a constrained angular aperture. However, it was shown in Paper I[6] that $42^{\circ} \pm \delta \theta$ for small $\delta \theta$ is not suitable for CE-based dosimetry due to practical considerations for detection. Instead, we consider the extreme large aperture case 
of $45^{\circ} \pm 45^{\circ}$. As also explained in Paper I, $90^{\circ}$ detection is simplest in terms of setup, allowing detector positioning in air with conventional phantom geometries. Due to the strong depth sensitivity near the surface and the beam quality dependence of $90^{\circ} \pm 5^{\circ}$ noted in Paper I, a large aperture of $90^{\circ} \pm 45^{\circ}$ is also considered in this case.

As explained in Paper I,[6] the code outputs dose per incident fluence and $k_{\mathrm{C}}^{\theta \pm \delta \theta}$ as energy deposited per CE photon spectral density at $n=1.34$ in units of $\mathrm{MeV} \cdot \mathrm{eV} \cdot$ photon $^{-1}$, from which we calculate CE photon spectral density per mass per incident fluence. It is trivial to change the value of the refractive index or incorporate its wavelength dependence in the code if deemed necessary. Unless otherwise specified, the default EGSnrc transport parameters were used.[16] The calculated values are therefore provisional, contingent on an investigation of the dependence on the fractional energy loss limit per step, ESTEPE. The scoring bins were cylindrical along the beam axis with radius of $1 \mathrm{~cm}$ and length of $0.05 \mathrm{~cm}$ near the surface and 0.1 or $0.2 \mathrm{~cm}$ for depths larger than $0.5 \mathrm{~cm}$ and nominal energies $<12 \mathrm{MeV}$ or $\geq 12 \mathrm{MeV}$, respectively. A sufficient number of histories, typically of the order of $10^{7}-10^{8}$ at incidence (of the order of 1-10 CPU hours, Intel Xeon E5-2687W), was simulated to ensure a Type A dose uncertainty at $d_{\max }$ within $0.3 \%$.

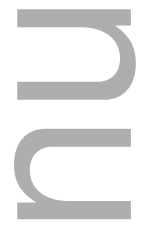

\section{RESULTS}

\section{A. Beam quality effects}

To ensure that the $k_{\mathrm{C}}^{\theta \pm \delta \theta}$ factor is representative of beam quality and correctly captures beam quality and depth variations as expected theoretically, we first examine the variation of the $k_{\mathrm{C}}^{90^{\circ} \pm 90^{\circ}}$ factor, i.e., $k_{\mathrm{C}}^{4 \pi}$, with beam quality and depth. At $\delta \theta<90^{\circ}$, the $k_{\mathrm{C}}^{\theta \pm \delta \theta}$ factor is further dependent on the $\mathrm{CE}$ signal anisotropy, which is also a function of beam quality and depth, and therefore beam quality and depth effects are more pronounced relative to $k_{\mathrm{C}}^{4 \pi}$.

Representative Monte Carlo percent-depth dose (PDD), percent-depth CE (PDC), and $k_{\mathrm{C}}^{4 \pi}$ results are shown in Figures 1 and 2. As in the work of Ding et al.[27] in the context of stopping powers for ion chamber dosimetry, the effects of the beam photon contamination, electron angular spread, and electron energy spread on $k_{\mathrm{C}}^{4 \pi}$ are separated for comparison as described in Table II. The "e- spectrum" data is not included in Figure 2 as it was found essentially equivalent to the "e- only" data as discussed below. The "photons-only" data is also shown for reference in Figure 1.

Due to the lack of CE below a certain threshold,[2] we also examined the effect of the CE threshold. The PDD, PDC, and contributions to the PDD of various portions of the beam quality with energies lower than the CE threshold are shown in Figure 3 for a $12 \mathrm{MeV}$ beam. Track ends, deltas, and particles with energies below the threshold at incidence ("incident") were found to contribute up to $8 \%, 1 \%$, and $0.5 \%$, respectively, to the PDD with all beam qualities studied. With the $\Delta$ cut-off set to a value of $10 \mathrm{keV}$,[24] well below the CE threshold of $257 \mathrm{keV}$,[2, 25] our calculated $k_{\mathrm{C}}^{\theta \pm \delta \theta}$ factors include below-threshold dose and, as we show below in Section III C, they are uniquely defined at each depth by the electron beam quality specifier, $R_{50}$, which can be obtained from Cherenkov measurements.

\section{B. Calculated $k_{\mathrm{C}}^{\theta \pm \delta \theta}$ factors}

In Figures 4 and 5 we show representative simulation results for the PDD, PDC generated in all directions $(4 \pi)$, and PDC generated at polar angles of $90^{\circ} \pm 5^{\circ}, 45^{\circ} \pm 45^{\circ}$, and $90^{\circ} \pm 45^{\circ}$ relative to the beam axis, as well as the

\section{This article is protected by copyright. All rights reserved}


corresponding PDD-to-PDC ratios. The latter are equivalent to CE-to-dose conversion factors, $k_{\mathrm{C}}^{\theta \pm \delta \theta}$ for $\theta \pm \delta \theta \in$ $\left\{90^{\circ} \pm 90^{\circ}(4 \pi), 90^{\circ} \pm 5^{\circ}, 45^{\circ} \pm 45^{\circ}, 90^{\circ} \pm 45^{\circ}\right\}$, normalized by the ratio of dose to CE maximum. Absolute $k_{\mathrm{C}}$ factors are made available through the normalization constants provided in the legend.

\section{Beam quality and reference depth specification in terms of $R_{50}$}

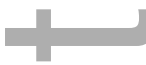

Here we address beam quality specification as well as the choice of a reference depth for CE-based dosimetry of electron beams. Because the $k_{\mathrm{C}}$ factor is a function of beam quality and depth, accurate CE-based dosimetry by the proposed method requires specification of both. Based on the Monte Carlo results of this study, we propose electron beam quality specification and examine the uncertainty at a CE-based reference depth in terms of the electron beam quality specifier $R_{50} \cdot[21]$

At each detector configuration, the Monte Carlo-calculated $R_{50}$ values, listed in Table I, were fit as a function of the depth of $50 \%$ CE $C_{50}$ (in units of $\mathrm{cm}$ ), selected from a linear interpolation of the PDC, with a second order polynomial, i.e.,

$$
R_{50}=A C_{50}^{2}+B C_{50}+C .
$$

The fit parameters for $4 \pi$ and $90^{\circ} \pm 5^{\circ}$ detection are $A=0.0003950 \mathrm{~cm}^{-1}, B=1.0138, C=0.1236 \mathrm{~cm}$, and $A=-0.00860 \mathrm{~cm}^{-1}, B=1.018, C=0.053 \mathrm{~cm}$, respectively. The fit root-mean-square deviation (RMSD) and maximum deviation are, respectively, $0.05 \mathrm{~mm}$ and $0.11 \mathrm{~mm}$ at $4 \pi$ and $0.20 \mathrm{~mm}$ and $0.33 \mathrm{~mm}$ at $90^{\circ} \pm 5^{\circ}$ detection. The fit RMSD and maximum deviation of the remaining configurations considered in Section III B are between these two extremes as one might expect. In addition, although exclusion of below-threshold[2] incident radiation would be expected to decrease the $R_{50}$ but not the $C_{50}$, at $4 \pi$ detection the difference between the full-beam and partialbeam $R_{50}$ was of the same order as the difference between the full-beam and partial-beam $C_{50}$, due to the statistical nature of the simulations, and the performance of the full-beam fit (Equation 2) on predicting the partial-beam $R_{50}$ was minimally affected $\left(\mathrm{RMSD}=0.06 \mathrm{~mm}\right.$ and maximum deviation $=0.19 \mathrm{~mm}$ ). The $90^{\circ} \pm 5^{\circ}$ fit, residuals, and performance on the experimental data of Paper I[6] are plotted in Figure 6 as a function of $C_{50}$.

In ionization-based reference dosimetry of electron beams according to AAPM's TG-51 protocol,[21] the reference depth $d_{\text {ref }}$ is given as a linear function of $R_{50}$. This ensures that $d_{\text {ref }}$ corresponds to $d_{\text {max }}$ for low-energy beams, avoiding uncertainties associated with corrections related to steep dose gradients, and optimizes the correlation of the stopping-power ratio with beam quality at $d_{\text {ref }}$ for high-energy beams.[28]

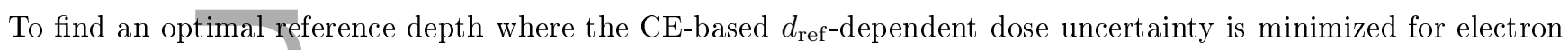
beams, based on our Monte Carlo study, the calculated $k_{\mathrm{C}}$ factor, cubic spline-interpolated at various beam qualitydependent as well as constant depths, was fit as a function of $R_{50}$ (in units of $\mathrm{cm}$ ) with an equation of the form

$$
k_{\mathrm{C}}\left(R_{50}, d_{\mathrm{ref}}\right)=A\left(R_{50}\right)^{B}+C\left(R_{50}\right)^{D} .
$$

The considered reference depths include the depth of maximum dose $d_{\max }$, constant depths at $1 \mathrm{~mm}$ intervals, and beam quality-dependent depths linear in $R_{50}$ (in units of $\mathrm{cm}$, listed in Table I), i.e.,

$$
d_{\mathrm{ref}}=A R_{50}+B
$$

\section{This article is protected by copyright. All rights reserved}



equipment and by adhering to recommended procedures.[29] To paint a quantitative picture of the uncertainty that could potentially be achievable by CE-based dosimetry, we estimate a preliminary uncertainty budget from our Monte Carlo and relative experimental results[6] at the optimal reference depth for electron beams (Section IIIC), as well as from approximations and best-case assumptions based on the available literature. The uncertainty budget, shown in Table IV, was evaluated according to the GUM protocol[30] from the expression for dose given in Equation 1, with the corrected reading expressed explicitly as in Equation 1a, and under the assumption that CE at the beam central axis is detected with a system which has been designed to have narrow and well-characterized response centered on the beam axis. As discussed in Paper I,[6] this could potentially be achieved via tomographic or optical sectioning techniques[8] and is a matter of further investigation.

A fit of the relative CE-to-dose conversion, $\mathrm{PD} k_{\mathrm{C}}$ (i.e., $\mathrm{PDD} / \mathrm{PDC}$ ), at the proposed $d_{\mathrm{ref}}$ for $90^{\circ} \pm 5^{\circ}$ acquisition (see Table III) was also carried out and its accuracy and performance on predicting the experimental $\mathrm{PD} k_{\mathrm{C}}$ data are plotted in Figure 7 . In part because $d_{\text {ref }}$ is selected to minimize the uncertainty of absolute $k_{\mathrm{C}}$ factors, the $\mathrm{PD} k_{\mathrm{C}}$ fit is less accurate, although it evidently performs well on the experimental data.[6] The larger deviations of the PDk $k_{\mathrm{C}}$ fit are also a result of the additional uncertainty contribution of the CE-to-dose maximum ratio. Furthermore, the $\mathrm{PD} k_{\mathrm{C}}$ fit was done on the ratio of PDD on the central axis to PDC within the optics acceptance volume (described in Paper I),[6] which varies more strongly with beam quality than central-axis PDD-to-PDC ratios.

\section{UNCERTAINTY BUDGET}

In ionization-based photon beam dosimetry, an uncertainty of $1 \%$ could be achievable with the use of calibrated techiques/8 and is a matter of further investigation.

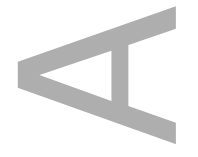

\section{A. Components of uncertainty}

1. Raw measured counts, $M_{\text {raw }}$

This is the raw reading, from which has been subtracted the background acquired during irradiation and with the optics input shielded. The Table IV value is the mean experimental robustness uncertainty contribution near $d_{\text {ref }}$ with 
a $12 \mathrm{MeV}$ TrueBeam electron beam (0.3\%), which was estimated for expected setup variations in Paper I,[6] and an additional $0.1 \%$ component is included in the $90^{\circ}$ case, which arises from a $0.01^{\circ}$ achievable optics angle tolerance.[6]

$$
\text { 2. } S S D, M_{\text {raw }}(\mathbf{S S D}, x, y, z, F S)
$$

In Table IV, a best-case uncertainty component of $M_{\text {raw }}$ due to the uncertainty of setting the SSD to $100 \mathrm{~cm}$ is assumed to be the same as that given in the TG-51 addendum.[29] The latter was calculated from the inverse square law.

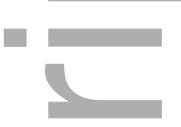

The positioning uncertainty contribution due to aligning the focal point of the objective at the central axis and at $d_{\text {ref }}$ will likely be dominated by the $d_{\text {ref }}$ positioning uncertainty, since the dose gradient is larger along the depth direction and relatively flat in the lateral direction. The $d_{\text {ref }}$ positioning uncertainty is envisioned to arise from setting the origin of the measurement system, which as in ion chamber dosimetry would constitute aligning the optical input at the water surface (demonstrated in Paper I),[6] as well as from the uncertainty of $R_{50}$ used in the calculation of $d_{\text {ref }}$ according to Equation 4. The Table IV values are evaluated as the product of the maximum dose gradient over all beam qualities studied normalized to local dose at $d_{\text {ref }}$ and a combined $d_{\text {ref }}$ uncertainty, which is based on a 0.5 $\mathrm{mm}$ uncertainty estimate of aligning the optics at the surface[29] as well as the $0.5 \mathrm{~mm}$ maximum error of our $R_{50}$ measurements (see Figure 6).[6] Note that the difference between the two detection configurations is of a statistical nature. For dose measurements at $R_{50}$, where the dose gradient is largest, the positioning uncertainty component due to a $0.5 \mathrm{~mm} R_{50}$ uncertainty is of the order of $10 \%$. Therefore, it is imperative that single-depth dose measurements are performed at a position of low dose gradient.

$$
\text { 4. Field size, } M_{\text {raw }}(S S D, x, y, z, \mathbf{F S})
$$

This is the uncertainty in $M_{\text {raw }}$ due to the uncertainty in setting the field size to $10 \times 10 \mathrm{~cm}^{2}$ or $20 \times 20 \mathrm{~cm}^{2}$ (see Section II A) and is evaluated as the product of typical values for the relative change in dose with field size and the field size uncertainty. Values for photon beams are provided by McEwen et al. (2014).[29] For electron beams, a similar variation with field size of approximately $1 \% / \mathrm{cm}$ at $R_{50}$ is typical[31] and is assumed in Table IV together with a field size uncertainty of $1 \mathrm{~mm}$.

\section{Temperature correction, $P_{\mathrm{T}}$}

This is the correction to the reading due to deviations of the water temperature from the reference temperature for which $k_{\mathrm{C}}(Q)$ values are calculated. The dominant effect is anticipated to be due to the variation of the index of refraction $n$ with temperature (0.03\%-0.04\% increase from $19{ }^{\circ} \mathrm{C}$ to $24^{\circ} \mathrm{C}$ at $200-1000 \mathrm{~nm}$ wavelengths, respectively)[25] and the corresponding variation in $M$ due to its dependence on $n$.[2] From the Frank-Tamm equation,[2] or equivalently

\section{This article is protected by copyright. All rights reserved}


from the CE power defined in Equation 1c of Paper I,[6] the contribution to $P_{\mathrm{T}}$ due solely to the CE signal variation with $n$ can be expressed as

$$
P_{\mathrm{T}}=\frac{1-\overline{\frac{1}{n\left(T_{0}\right)^{2}}}}{1-\frac{1}{\frac{1}{\beta^{2}}}},
$$

where $T_{0}$ is the reference temperature, $T$ is the water temperature at the time of measurement, $\beta$ is the charged particle velocity normalized to the speed of light in vacuum, and the two averaged terms are over the CE acquisition bandwidth and the charged particle fluence spectrum, respectively. For variations on the order of a few degrees near room temperature and acquisition bandwidth of $200 \mathrm{~nm}$ to $1000 \mathrm{~nm}, P_{\mathrm{T}}$ corresponds to corrections of $0.07 \%$ to $0.1 \%$, respectively, for relativistic electrons and $0.04 \%$ for electron energies near the CE threshold.[25] In addition, the $\mathrm{CE}$ angle variation[3] due to variations of a few degrees at room temperature and for the same wavelength range is at most $0.02^{\circ}$ for relativistic electrons. If taken into account, the uncertainty of this correction will be evaluated from the uncertainty of $n$. If ignored due to its small magnitude (i.e., setting $P_{\mathrm{T}}=1.000$ ), a Type $\mathrm{B}$ uncertainty can be assigned based on its magnitude. The Table IV value is based on a rectangular distribution on a $0.1 \%$ correction.

\section{Calibration, $N$}

The specifics of the calibration procedure and corresponding uncertainty require detector development and characterization via optical design and are therefore a matter of further investigation. Here we estimate a provisional calibration uncertainty by considering that the entire detection system is calibrated in the clinic with a calibration light source traceable to a primary standards lab. The uncertainty of the calibration coefficient $N$ then arises from the uncertainty of the light source calibration and the transfer of the calibration to the optical detector system. The standard uncertainty $(k=1)$ of the primary luminous intensity scale of the National Research Council, Canada, is $0.5 \%$. For lack of a better approximation at this stage, we assume a transfer uncertainty of the same order, which leads to a combined provisional estimate of $0.7 \%$ in Table IV. This is potentially the largest source of uncertainty of our proposed method.

\section{Calibration stability, $N$ stability}

The stability of the calibration will depend on the stability of the calibration light source if the system is calibrated at the time of measurement, or on the stability of the optical detector system if it is calibrated in advance. The corresponding stability in either case is dependent on the particular light source or detector used and must be monitored by the user. The Table IV value corresponds to the light ripple specification of calibrated light sources supplied by Newport Corporation.

\section{CE-to-dose conversion, $k_{\mathrm{C}}$}

This component is due to the relative combined standard uncertainty of the Monte Carlo-calculated $k_{\mathrm{C}}(Q)$ values. Its complete evaluation is a matter of further investigation and would include the statistical uncertainty of the

\section{This article is protected by copyright. All rights reserved}


calculated values, uncertainty of the index of refraction, uncertainty of the approximations used in EGSnrc, and uncertainties in the interaction cross sections used in the simulation. The overall Type B uncertainty can be estimated by looking at the difference between calculation and measurement. Table IV values are based on a rectangular distribution on the maximum difference (over all beam qualities studied) between simulated and experimental relative $k_{\mathrm{C}}$ factors $(0.3 \%$, although a conservative estimate was made of $0.5 \%)$, as well as the RMSD Monte Carlo statistical uncertainty of simulated $k_{\mathrm{C}}$ factors $\left(0.07 \%\right.$ and $0.3 \%$ for $4 \pi$ and $90^{\circ} \pm 5^{\circ}$ detection, respectively) and the Type A uncertainty of relative $k_{\mathrm{C}}$ measurements with $2^{\circ}$ angular aperture $(0.5 \%)$ at $d_{\text {ref. }}[6]$

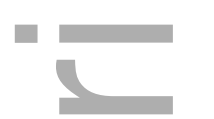

9. $k_{\mathrm{C}}$ assignment

The uncertainty due to the assignment of the $k_{\mathrm{C}}(Q)$ factor at $d_{\text {ref }}$ includes contributions from the beam quality specifier $Q$ and interpolation of the $k_{\mathrm{C}}(Q)$ data to the measured $Q$. The latter may constitute the variation of $k_{\mathrm{C}}(Q)$ with $Q$ in the case of interpolation from neighboring data points or the data fit uncertainty if a fit is used for interpolation. Because this value depends on the beam quality and the chosen method of interpolation, it must be determined by the user. Table IV values are based on the RMSD of the $k_{\mathrm{C}}\left(R_{50}\right)$ fit (Equation 3 ) and the uncertainty of the assigned $k_{\mathrm{C}}\left(R_{50}\right)$ value due to the RMSD of the $R_{50}\left(C_{50}\right)$ fit (Equation 2$)$.

10. Linac stability

This component accounts for the short-term repeatability of the machine output for a fixed measure of the beam-on time. The value in Table IV is that reported by McEwen et al.[29] based on a review of the literature on the stability of modern linacs.

\section{B. Combined dose uncertainty}

Our baseline estimate of the combined standard uncertainty of $D(Q)$, based on the stated assumptions, approximations, and assuming that all components are uncorrelated, is on the order of $1 \%$. As in the case of ionization-based dosimetry, determining the achievable level of uncertainty with CE-based dosimetry will require a great deal of research effort and collaboration.[29] Here we provide a starting point by calculating an approximate ballpark of the achievable uncertainty from our Monte Carlo and relative experimental results with a simple detector design[6] as well as from best-case assumptions and approximations based on the available literature.

\section{DISCUSSION}

With the aim of bringing CE-based dosimetry of external radiotherapy beams to routine clinical use, we have Monte Carlo-calculated provisional electron beam CE-to-dose conversion factors, $k_{\mathrm{C}}^{\theta \pm \delta}$, for a clinically representative library of beam qualities to be used with the formalism presented in the companion Paper I, which also provides supportive experimental results.[6] The simulations presented here are validated through the relative experimental study of Paper I. In Section III C, we address beam quality specification as well as the choice of a reference depth

\section{This article is protected by copyright. All rights reserved}


for CE-based dosimetry of electron beams. In Section IV, based on the Monte Carlo and experimental results of the current study and accompanying Paper I, we then estimate a best-case preliminary uncertainty budget for CE-based dose measurement at a representative clinically-relevant reference depth.

The ratio of stopping power to $\mathrm{CE}$ power[6] in water is $\sim 10 \%$ greater for $1 \mathrm{MeV}$ electrons compared to $20 \mathrm{MeV}$ electrons. That is, the $4 \pi$ CE-to-dose ratio of electron beams decreases with electron energy and increases with depth. We first take a look at the depth dependence of the full beam CE generated within a $4 \pi$ solid angle and the corresponding dose-to-CE conversion in Section III A. For any poly- or mono-energetic electron beam in the therapeutic energy range, we have observed that the PDC is upstream of the PDD (see Figures 1 and 2) due to the lack of $\mathrm{CE}$ contribution from the portion of the charged particle fluence spectrum lower than the CE threshold (260 keV in water at $500 \mathrm{~nm}) .[2,25]$ This effect was also observed and briefly discussed in a previous study.[12] The upstream shift is equal to the projected distance along the depth direction over which charged particles lose 260 keV. The discrepancy is more pronounced near the surface because of smaller mean scattering angles and therefore longer charged particle projected ranges. For high-energy charged particles, this distance is in the range of 1-2 mm at larger depths.[32] Since at high energies stopping power and CE power are both only weakly dependent on energy, dose deposition and CE generation by high-energy beams are mainly determined by the total fluence and less by the spectral distribution of the fluence. The total fluence generating CE, i.e., with energy $>260 \mathrm{keV}$, is the same as the total fluence depositing dose downstream at a distance of 1-2 mm at high energies. Quantitatively, for electrons of energy $E$, mass restricted stopping power $L(E)$, and energy loss $E_{\mathrm{thr}}=260 \mathrm{keV}$, the CSDA approximation of this distance is $-\int_{E}^{E-E_{\mathrm{thr}}} d E^{\prime} / L\left(E^{\prime}\right) \approx E_{\mathrm{thr}} / L(E)$, which is 1.1-2.2 mm for $E>300 \mathrm{keV}$ and cut-off energy $\Delta=1-100 \mathrm{keV}$ in water.[32]

We now turn our attention to beam quality effects on $k_{\mathrm{C}}^{4 \pi}$, which were evaluated as illustrated in Table II. Although lower-energy electrons are scattered at larger angles and are therefore expected to increase $k_{\mathrm{C}}^{4 \pi}$ near the surface, angular spread appears to have a minimal effect on $k_{\mathrm{C}}^{4 \pi}$, as seen by the small difference between the "e- only" and "espectrum" curves ( $\leq \sim 0.2 \%$ of "full beam" at $\left.d_{\max }\right)$ in Figure 1 . For this reason, the "e- spectrum" curve was excluded from Figure 2. In addition, since high-energy radiation is more deeply penetrating, a low-energy component of the beam spectrum increases $k_{\mathrm{C}}^{4 \pi}$ at shallow depths and a high-energy component decreases $k_{\mathrm{C}}^{4 \pi}$ at large depths. Therefore, the "full beam" $k_{\mathrm{C}}^{4 \pi}$ exhibits less variation with depth than the corresponding "mono-energetic" $k_{\mathrm{C}}^{4 \pi}$ due both to the energy spread of photon contamination ("full beam" versus "e- only" in Figures 1 and 2) and the electron energy spread ("e- spectrum" or "e- only" versus "mono-energetic"). For the lowest-energy beams (Figure 2), the electron energy spread has the largest effect on $k_{\mathrm{C}}^{4 \pi}$ in the build-up and at $d_{\max }(\leq \sim 0.8 \%$ of "full beam"), giving way to the effect of photon contamination ( $\leq \sim 3 \%$ of "full beam" at $\left.d_{\max }\right)$ with increasing beam energies (Figure 1). As one might expect, $k_{\mathrm{C}}^{4 \pi}$ of all beams is largely determined by the electron energy spread in the drop-off region and by photon contamination in the bremsstrahlung tail.

Comparing machines from the same manufacturer, similar results were obtained with the Clinac 2100C/D (Figure

2) and 21EX (not shown for this reason). Meanwhile, the TrueBeam has a broader electron spectrum, due to its different scattering foil geometry, which has a notably larger effect on $k_{\mathrm{C}}^{4 \pi}$ in the drop-off region ("e- only" versus "mono-energetic" in Figure 2). Comparing manufacturers, the effect of the lower photon contamination of the Elekta Precise relative to the Varian machines was also readily observed as a decrease in the separation between the "full beam" and "e- only" $k_{\mathrm{C}}^{4 \pi}$ (see Figure 1 for the TrueBeam case). Because of the non-negligible effect of beam quality,

\section{This article is protected by copyright. All rights reserved}


from here onward we perform full beam simulations.

In contrast to the $4 \pi$ PDC, the PDC at polar angles of $\theta \pm \delta \theta$ for small $\delta \theta$ depends on the scattering of the beam due to the anisotropy of CE.[3] Therefore, as in the TrueBeam case of the accompanying Paper I,[6] the PDC at $90^{\circ} \pm 5^{\circ}$ in Figures 4 and 5 is characterized by a steeper build-up and larger depth of maximum CE than the $4 \pi$ PDC. On the other hand, the larger two considered $\pm 45^{\circ}$ apertures more closely resemble the $4 \pi$ case, with the PDC at $45^{\circ} \pm 45^{\circ}$ appearing upstream of the $4 \pi \mathrm{PDC}$ as it includes the $42^{\circ} \mathrm{CE}$ angle of relativistic electrons in water[3, 25] and it generally corresponds to higher-energy more forward-directed charged particles. It is evident in Figures 4 and 5 that the reduced depth and beam quality dependence of $k_{\mathrm{C}}^{4 \pi}$ could be attained with a large aperture at an intermediate $\theta$ value to $45^{\circ}$ and $90^{\circ}$ without the requirement of $4 \pi$ detection. Referring to Figure $12 \mathrm{~b}$ of Paper I[6] reveals that this could entail a symmetric integration of the $\mathrm{CE}$ angular distribution (e.g., over its full width at half maximum at a given depth).

In Section IIIC, the $R_{50}\left(C_{50}\right)$ fit RMSD and maximum deviation, ranging from $0.05 \mathrm{~mm}$ and $0.11 \mathrm{~mm}$ at $4 \pi$ to $0.20 \mathrm{~mm}$ and $0.33 \mathrm{~mm}$ at $90^{\circ} \pm 5^{\circ}$ detection, respectively, is on par with ionization-based dosimetry, where $R_{50}$ is estimated from a fit in terms of the depth of $50 \%$ ionization $I_{50}$ to within $0.4 \mathrm{~mm}$.[21, 27] The $k_{\mathrm{C}}\left(R_{50}, d_{\mathrm{ref}}\right)$ fit RMSD and maximum deviation of $0.42 \%$ and $0.89 \%$ at $4 \pi$ and $0.78 \%$ and $2.0 \%$ at $90^{\circ} \pm 5^{\circ}$ detection, respectively, can be improved by fitting low and high-energy beams separately as is done for electron beam quality specification in ionization-based dosimetry.[21] Alternatively or in addition to this, a downstream shift of the $4 \pi$ PDC can be performed in order to improve the correlation with the PDD. It is important to note that with the default ESTEPE value of $0.25[16]$ our Monte Carlo calculations of the TrueBeam $20 \mathrm{MeV} k_{\mathrm{C}}^{90^{\circ} \pm 5^{\circ}}$ near $d_{\text {ref }}$ were $1.1 \% \pm 0.3 \%$ lower relative to an equivalent single scattering simulation, while in the $4 \pi$ case this difference was zero within uncertainties. It is therefore rooted in the assumption of a straight transport step in scoring CE within a constrained aperture.[6] The small-aperture calculations are therefore provisional and contingent on a full ESTEPE investigation and code optimization in the case that CE-based dosimetry will involve small apertures instead of the large apertures envisioned in this study.

As explained at the end of Section III A, the PDD contribution of portions of the beam quality of energies below the $\mathrm{CE}$ threshold is included in the calculated CE-to-dose conversion for our library of clinically representative electron beam models. The value of the conversion can be interpolated to the clinical beam quality as shown in Section III C. For differences between the user beam and the interpolated conversion arising solely from exclusion (or equivalently doubling) of the portion of the incident beam spectrum below the $\mathrm{CE}$ threshold, the $R_{50}$ value obtained from a fit in terms of $C_{50}$ will be the same (Section IIIC) and the dose error relative to the dose at $d_{\max }$ will be at most $0.5 \%$ (Section III A).

It becomes apparent from the results of this study that the value of the electron beam CE-to-dose conversion, $k_{\mathrm{C}}$, is a function of both beam quality and depth. This is also the case for the electron beam water-to-air stopping ratio used in ionization-based dosimetry[27, 28] as well as for the electron beam quality conversion factor, $k_{Q}$, which is analogous to $k_{\mathrm{C}}$ at depth $d_{\text {ref }}$ in ionization-based reference dosimetry.[21] The $k_{Q}$ factor is also a function of ionization chamber type and make.

As defined in the AAPM TG-51 protocol,[21] $k_{Q}=P_{\mathrm{gr}}^{Q} k_{R_{50}}$. The gradient correction factor, $P_{\mathrm{gr}}^{Q}$, involves a shift of the percent-depth ionization (PDI) to correct for the fluence gradient across the ionization chamber volume and to better match the PDD in water. This may also potentially be done in CE-based dosimetry, where the PDC will be 
shifted downstream to better match the PDD by the difference between $R_{50}$, estimated as in Equation 2, and $C_{50}$.

The water-to-air stopping-power ratio variation with depth up to $R_{50}$ for clinical electron beam energies of $9 \mathrm{MeV}$ and higher can be up to $\pm 10 \%$.[27] The variation of the $4 \pi k_{\mathrm{C}}$ with depth up to $R_{50}$, before shifting the PDC, for energies of $4 \mathrm{MeV}$ and higher is up to $\pm 24 \%$ ( $\pm 17 \%$ for energies of $9 \mathrm{MeV}$ and higher). If the PDC is shifted, this variation decreases to $\pm 2.8 \%$. The variation of $k_{R_{50}}$ (i.e., after shifting the PDI) with beam quality specifier $R_{50}$ of $2-9 \mathrm{~cm}$, at the electron beam reference depth for ionization-based dosimetry, is up to $\pm 2 \%$ to $\pm 3 \%$, depending on ionization chamber type and make.[21] The corresponding variation of the $4 \pi k_{\mathrm{C}}$, without a PDC shift, is up to $\pm 3.0 \%$ and with a shift it is up to $\pm 2.8 \%$.

The beam quality and depth dependence of the electron beam $4 \pi k_{\mathrm{C}}$ (before shifting the PDC) is therefore of the same order as the conversions used in ionization-based electron beam dosimetry and potentially better if a shift is applied. For smaller angular apertures than $4 \pi$, the variation is higher. A larger numerical aperture detector will therefore be preferable.

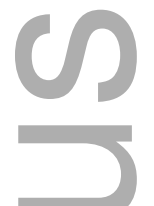

\section{CONCLUSIONS}

In this study, we pave the way towards possible clinical implementation of CE-based external beam dosimetry by Monte Carlo-calculating provisional broad-beam central-axis CE-to-dose conversion factors in water, considering detection configurations, beam quality specification, and reference depth selection, and estimating a preliminary uncertainty budget for electron beams of the order of $1 \%$. This is motivated and facilitated by the formalism, code design, and relative experimental validation of an accompanying Paper I.[6] It is our hope that these findings will inspire and inform future efforts toward dedicated optical design and characterization of a CE-based detector with narrow response on beam axis, which may involve optical sectioning[8] or tomographic methods. Energy spectra and angular distributions of CE, presented in Paper I, can provide necessary input into the optical design platform. With advances in CE-based detector development, potentially building upon the 3D reconstruction techniques already available,[4, 5, 10] clinical implementation of CE-based electron beam dosimetry is now within reach.

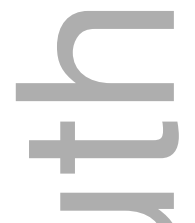

\section{ACKNOWLEDGMENTS}

The authors would like to thank Tanner Connell of McGill University for providing beam models, clinical data, and guidance, Joe Larkin of the McGill University Health Centre for help with construction of the experimental setup, Francois Therriault-Proulx of Centre Hospitalier Universitaire de Montréal for valuable discussions on optical instrumentation, and Marc-André Renaud for help with setting up the simulations. YZ acknowledges partial support by the CREATE Medical Physics Research Training Network grant of the Natural Sciences and Engineering Research Council (NSERC) (\#432290), CREATE - Integrated Sensor Systems grant of NSERC (\#371305), and Bourses de doctorat en recherche (B2) du Fonds de recherche du Québec - Nature et technologies (FRQNT) (\#184385). This work was partially supported by NSERC (\#RGPIN-2014-06475, \#231909).

\section{This article is protected by copyright. All rights reserved}




\section{DISCLOSURE OF CONFLICTS OF INTEREST}

The authors have no conflicts to disclose.

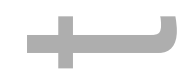

[1] P. Cherenkov, "Vidimoe svechenie chistykh zhidkostei pod deistviem gamma-radiatsii" (Visible glow of pure liquids under gamma radiation), Dokl. Akad. Nauk SSSR 2, 451-457 (1934).

[2] I. Tamm and I. Frank, "Kogerentnoe izluchenie bystrogo elektrona v srede" (Coherent radiation of a fast electron in a medium), Dokl. Akad. Nauk 14, 107-112 (1937).

[3] J. V. Jelley, Cerenkov radiation and its applications, British Journal of Applied Physics 6, 227-232 (1955).

[4] A. K. Glaser, J. M. Andreozzi, R. Zhang, B. W. Pogue, and D. J. Gladstone, Optical cone beam tomography of Cherenkovmediated signals for fast 3D dosimetry of X-ray photon beams in water, Med Phys 42, 4127-4136 (2015).

[5] P. Bruza, J. M. Andreozzi, D. J. Gladstone, L. A. Jarvis, J. Rottmann, and B. W. Pogue, Online Combination of EPID \& Cherenkov Imaging for 3-D Dosimetry in a Liquid Phantom, IEEE Transactions on Medical Imaging 36, 2099-2103 (2017).

[6] Y. Zlateva, B. Muir, I. El Naqa, and J. Seuntjens, Cherenkov emission-based external radiotherapy dosimetry: I. Formalism and feasibility, Med Phys (In Print).

[7] D. Malacara-Hernández and Z. Malacara-Hernández, Handbook of Optical Design, Third Ed., CRC Press, 2014.

[8] D. A. Agard, Y. Hiraoka, P. Shaw, and J. W. Sedat, Chapter 13 Fluorescence Microscopy in Three Dimensions, in Fluorescence Microscopy of Living Cells in Culture Part B. Quantitative Fluorescence Microscopy-Imaging and Spectroscopy, edited by D. L. Taylor and Y.-L. Wang, volume 30 of Methods in Cell Biology, pages 353 - 377, Academic Press, 1989.

[9] A. K. Glaser, S. C. Davis, D. M. McClatchy, R. Zhang, B. W. Pogue, and D. J. Gladstone, Projection imaging of photon beams by the Čerenkov effect, Med Phys 40, 012101-1-012101-13 (2013).

[10] A. K. Glaser, W. H. A. Voigt, S. C. Davis, R. Zhang, D. J. Gladstone, and B. W. Pogue, Three-dimensional Cerenkov tomography of energy deposition from ionizing radiation beams, Opt. Lett. 38, 634-636 (2013).

[11] Y. Helo, I. Rosenberg, D. D’Souza, L. MacDonald, R. Speller, G. Royle, and A. Gibson, Imaging Cerenkov emission as a quality assurance tool in electron radiotherapy, Phys Med Biol 59, 1963-1978 (2014).

[12] A. K. Glaser, R. Zhang, D. J. Gladstone, and B. W. Pogue, Optical dosimetry of radiotherapy beams using Cherenkov radiation: the relationship between light emission and dose, Phys Med Biol 59, 3789-3811 (2014).

[13] D. W. O. Rogers, B. A. Faddegon, G. X. Ding, C.-M. Ma, J. We, and T. R. Mackie, BEAM: A Monte Carlo code to simulate radiotherapy treatment units, Med Phys 22, 503-524 (1995).

[14] D. Rogers, B. Walters, and I. Kawrakow, BEAMnrc Users Manual, Technical Report PIRS-0509(A)revL, National Research Council of Canada, Ottawa, Canada, 2012.

[15] I. Kawrakow, Accurate condensed history Monte Carlo simulation of electron transport. I. EGSnrc, the new EGS4 version, Med Phys 27, 485-498 (2000).

[16] I. Kawrakow, E. Mainegra-Hing, D. Rogers, F. Tessier, and B. Walters, The EGSnrc Code System: Monte Carlo Simulation of Electron and Photon Transport, Technical Report PIRS-701, National Research Council of Canada, Ottawa, Canada, 2011.

[17] L. Brualla, R. Palanco-Zamora, A. Wittig, J. Sempau, and W. Sauerwein, Comparison between PENELOPE and electron Monte Carlo simulations of electron fields used in the treatment of conjunctival lymphoma, Phys Med Biol 54, 5469-5481 (2009).

\section{This article is protected by copyright. All rights reserved}


[18] T. Connell, A. Alexander, M. Evans, and J. Seuntjens, An experimental feasibility study on the use of scattering foil free beams for modulated electron radiotherapy, Phys Med Biol 57, 3259-3272 (2012).

[19] A. Rodrigues, D. Sawkey, F.-F. Yin, and Q. Wu, A Monte Carlo simulation framework for electron beam dose calculations using Varian phase space files for TrueBeam Linacs, Med Phys 42, 2389-2403 (2015).

[20] B. R. Muir and D. W. O. Rogers, Monte Carlo calculations for reference dosimetry of electron beams with the PTW Roos and NE2571 ion chambers, Med Phys 40, 121722 (2013).

[21] P. R. Almond, P. J. Biggs, B. M. Coursey, W. F. Hanson, M. S. Huq, R. Nath, and D. W. O. Rogers, AAPM's TG-51 protocol for clinical reference dosimetry of high-energy photon and electron beams, Med Phys 26, 1847-1870 (1999).

[22] https://github.com/yanazlateva/scrrznrc/tree/f5356f63166a538ebd3b8f84775b5de69e895428.

[23] D. Rogers, I. Kawrakow, J. Seuntjens, B. Walters, and E. Mainegra-Hing, NRC User Codes for EGSnrc, Technical Report PIRS-702(RevB), National Research Council of Canada, Ottawa, Canada, 2003.

[24] L. V. Spencer and F. H. Attix, A Theory of Cavity Ionization, Radiation Research 3, 239-254 (1955).

[25] M. Daimon and A. Masumura, Measurement of the refractive index of distilled water from the near-infrared region to the ultraviolet region, Appl. Opt. 46, 3811-3820 (2007).

[26] G. M. Hale and M. R. Querry, Optical Constants of Water in the 200-nm to 200- $\mu$ m Wavelength Region, Appl. Opt. 12, $555-563$ (1973).

[27] G. X. Ding, D. W. O. Rogers, and T. R. Mackie, Calculation of stopping-power ratios using realistic clinical electron beams, Med Phys 22, 489-501 (1995).

[28] D. T. Burns, G. X. Ding, and D. W. O. Rogers, R50 as a beam quality specifier for selecting stopping-power ratios and reference depths for electron dosimetry, Med Phys 23, 383-388 (1996).

[29] M. McEwen, L. DeWerd, G. Ibbott, D. Followill, D. W. O. Rogers, S. Seltzer, and J. Seuntjens, Addendum to the AAPM's TG-51 protocol for clinical reference dosimetry of high-energy photon beams, Med Phys 41, 041501-1-041501-20 (2014), 041501.

[30] Evaluation of measurement data - Guide to the expression of uncertainty in measurement, Technical Report JCGM 100:2008 - GUM 1995 with minor corrections, International Organization for Standardization, Geneva, Switzerland, 2008.

[31] E. Podgoršak, editor, Radiation Oncology Physics: A Handbook for Teachers and Students, pages 273-286, STI/PUB, International Atomic Energy Agency, 2005.

[32] Stopping Powers for Electrons and Positrons, Technical Report 37, International Commission on Radiation Units and Measurements, 1984.

TABLE I. BEAMnrc[13, 14] models, nominal electron beam energies, and beam quality specifier $R_{50}$.

\begin{tabular}{ccc}
\hline \hline Model & Nominal energies / MeV & $R_{50} / \mathrm{cm}$ \\
\hline Clinac 2100C/D & 6,9 & $2.531,3.769$ \\
Clinac 21EX & $6,9,12,16,20$ & $2.533,3.718,5.170,6.819,8.481$ \\
TrueBeam & $6,9,12,15,16,18,20,22$ & $2.302,3.509,4.943,6.195,6.594,7.540,8.240,8.816$ \\
Precise & $4,8,12,18,22$ & $1.785,3.286,4.933,7.051,8.832$ \\
\hline
\end{tabular}


TABLE II. Beam quality effects were examined by calculating and comparing $4 \pi$ CE-to-dose conversion factors, $k_{\mathrm{C}}^{4 \pi}$, of various portions of the beam quality as described here, with the "<" symbol designating the comparison. The "photons only" $k_{\mathrm{C}}^{4 \pi}$ is also calculated for reference.

\begin{tabular}{|c|c|c|}
\hline Beam quality factor & $k_{\mathrm{C}}^{4 \pi}$ calculation & Description \\
\hline $\begin{array}{r}\text { photon contamination } \\
\text { angular spread } \\
\text { energy spread }\end{array}$ & $\begin{array}{l}\text { full beam } \\
\text { e- only } \\
\text { e- spectrum } \\
\text { mono-energetic } \\
\text { photons only }\end{array}$ & $\begin{array}{l}\text { all particles of the beam } \\
\text { only the electrons of the beam } \\
\text { electron point source at } 100-\mathrm{cm} \text { SSD, same spectrum as "e- only" on axis at } z=0 \\
\text { mono-energetic parallel electrons at the beam central-axis mean incident energy } \\
\text { only the contaminant photons of the beam }\end{array}$ \\
\hline
\end{tabular}

TABLE III. Reference depth $d_{\text {ref }}$ according to Equation $4, k_{\mathrm{C}}$ fit parameters according to Equation 3 , and $k_{\mathrm{C}}$ factors at $d_{\text {ref }}$ for selected values of the electron beam quality specifier $R_{50}$ for $4 \pi$ and $90^{\circ} \pm 5^{\circ}$ detection.

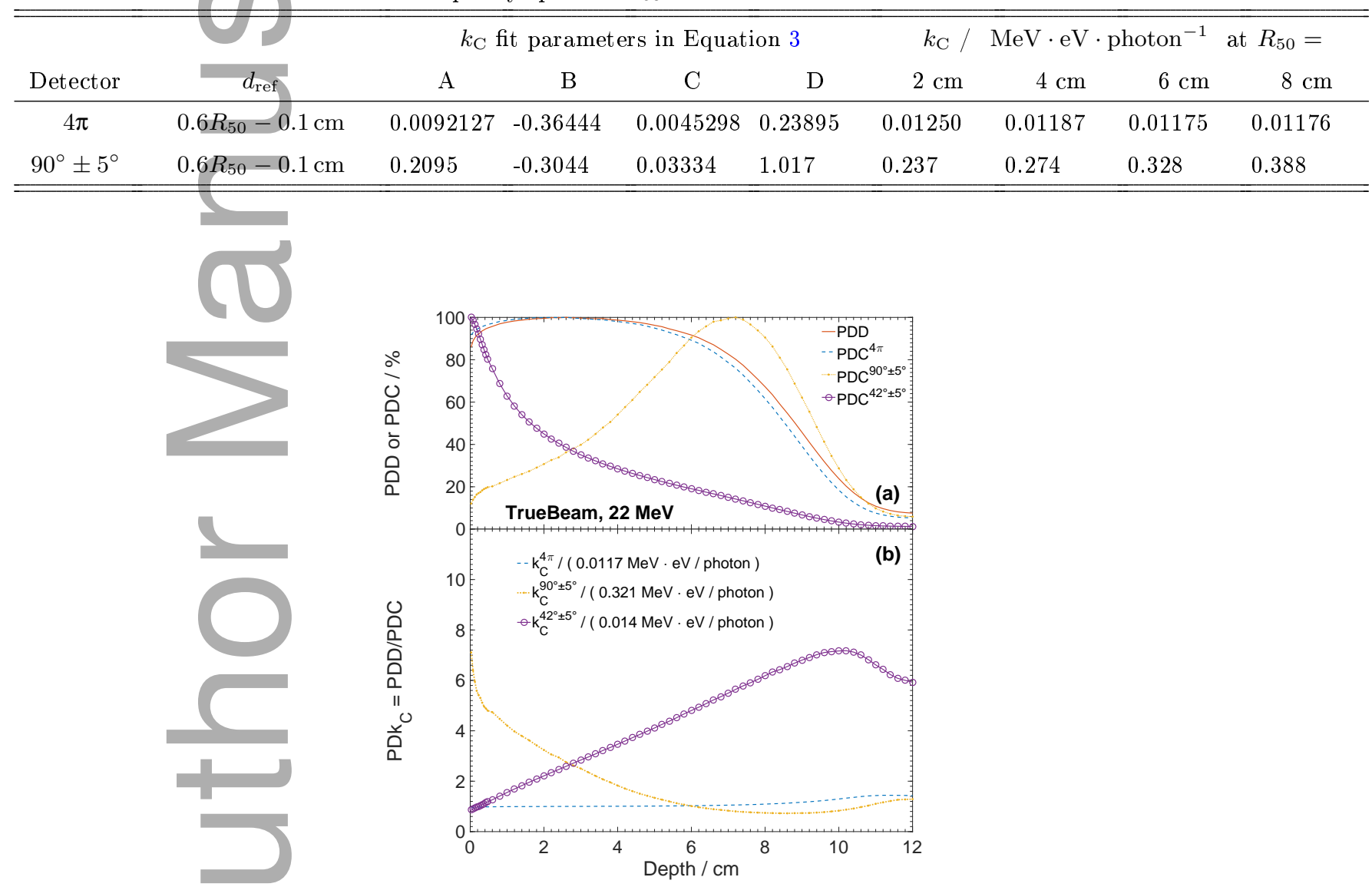

FIG. 1. Simulated (a) percent-depth dose, PDD, percent-depth Cherenkov emission (CE), PDC, and (b) CE-to-dose conversion factor for CE generated within a $4 \pi$ solid angle, $k_{\mathrm{C}}^{4 \pi}$, in water for a TrueBeam $22 \mathrm{MeV}$ electron beam. Beam quality effects on $k_{\mathrm{C}}^{4 \pi}$ are separated for comparison as described in Table II. 
TABLE IV. Preliminary best-case uncertainty budget of the proposed Cherenkov emission-based formalism (Paper I[6] and Equation 1) for the measurement of absorbed dose to water in electron beams at an optimal depth $d_{\text {ref }}$ (Section III C) under reference conditions. Estimates are given as relative standard uncertainties $(k=1)$. Detector angles listed are relative to the beam direction and correspond to the same number of histories. The combined estimate assumes all components are uncorrelated.

\begin{tabular}{|c|c|c|c|}
\hline Component & Section & $\begin{array}{c}4 \pi \text { detection } \\
d_{\mathrm{ref}}=0.6 R_{50}-0.1 \mathrm{~cm}\end{array}$ & $\begin{array}{c}90 \pm \delta \theta, \delta \theta \leq \mathbf{5}, \text { detection } \\
d_{\mathrm{ref}}=0.6 R_{50}-0.1 \mathrm{~cm}\end{array}$ \\
\hline \multicolumn{4}{|l|}{ Measurement } \\
\hline$M_{\text {raw }}$ & IV A 1 & $0.3 \%$ & $0.3 \%$ \\
\hline$S S D$ & IV A 2 & $0.10 \%$ & $0.10 \%$ \\
\hline$x, y, z$ & IV A 3 & $0.39 \%$ & $0.46 \%$ \\
\hline$F S$ & IV A 4 & $0.10 \%$ & $0.10 \%$ \\
\hline$P_{\mathrm{T}}$ & IV A 5 & $0.06 \%$ & $0.06 \%$ \\
\hline \multicolumn{4}{|l|}{ Calibration } \\
\hline$N$ & IV A 6 & $0.7 \%$ & $0.7 \%$ \\
\hline$N$ stability & IV A 7 & $0.1 \%$ & $0.1 \%$ \\
\hline \multicolumn{4}{|l|}{ Conversion } \\
\hline$k_{\mathrm{C}}$ & IV A 8 & $0.56 \%$ & $0.58 \%$ \\
\hline$k_{\mathrm{C}}$ assignment & IV A 9 & $0.43 \%$ & $0.80 \%$ \\
\hline \multicolumn{4}{|l|}{ Influence quantities } \\
\hline Linac stability & IV A 10 & $0.05 \%$ & $0.05 \%$ \\
\hline COMBINED $(k=1)$ & IV B & $1.1 \%$ & $1.3 \%$ \\
\hline$R_{50}\left(C_{50}\right)$ fit RMSD & III C & $0.05 \mathrm{~mm}$ & $0.20 \mathrm{~mm}$ \\
\hline
\end{tabular}



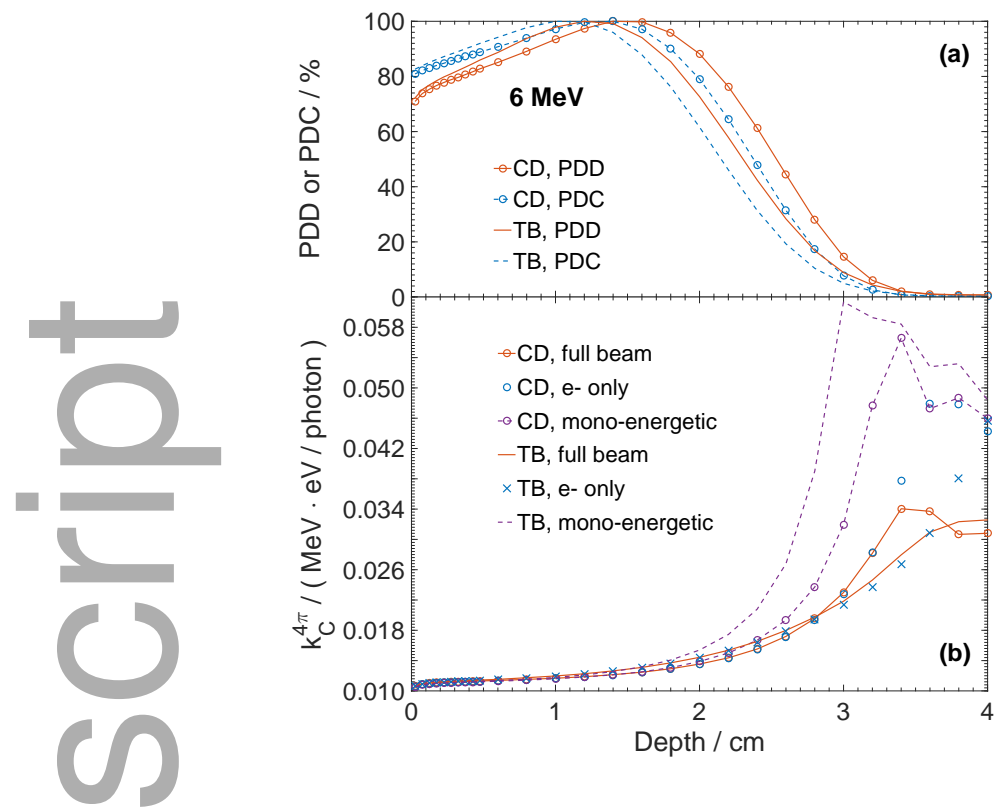

FIG. 2. Same as Figure 1 but for Clinac 2100C/D ("॰”, CD) and TrueBeam (TB) 6 MeV electrons, excluding the "photons only" and "e- spectrum" curves.

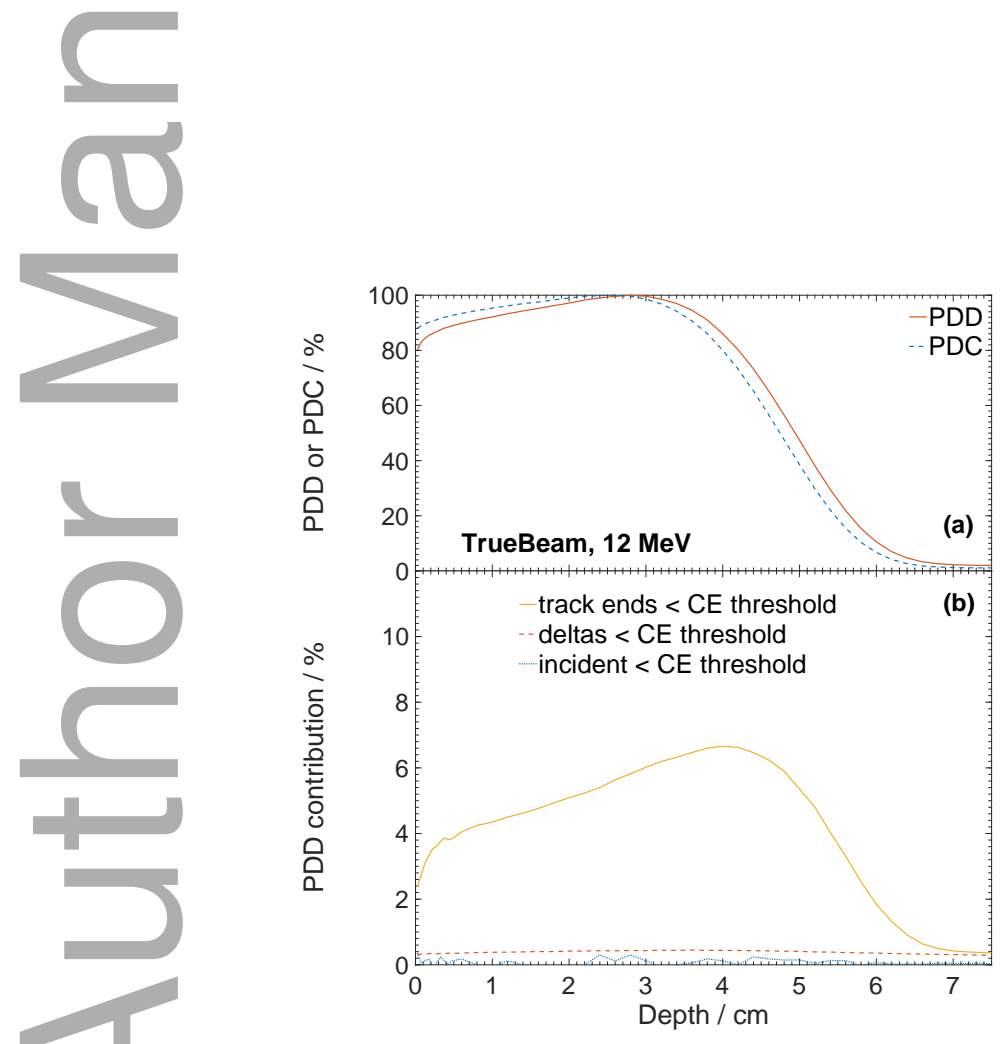

FIG. 3. Simulated (a) percent-depth dose, PDD, percent-depth Cherenkov emission, PDC, and (b) contributions to the PDD of various portions of the beam quality with energies lower than the Cherenkov emission (CE) threshold in water (260 keV)[2, 25] for a TrueBeam $12 \mathrm{MeV}$ electron beam. 

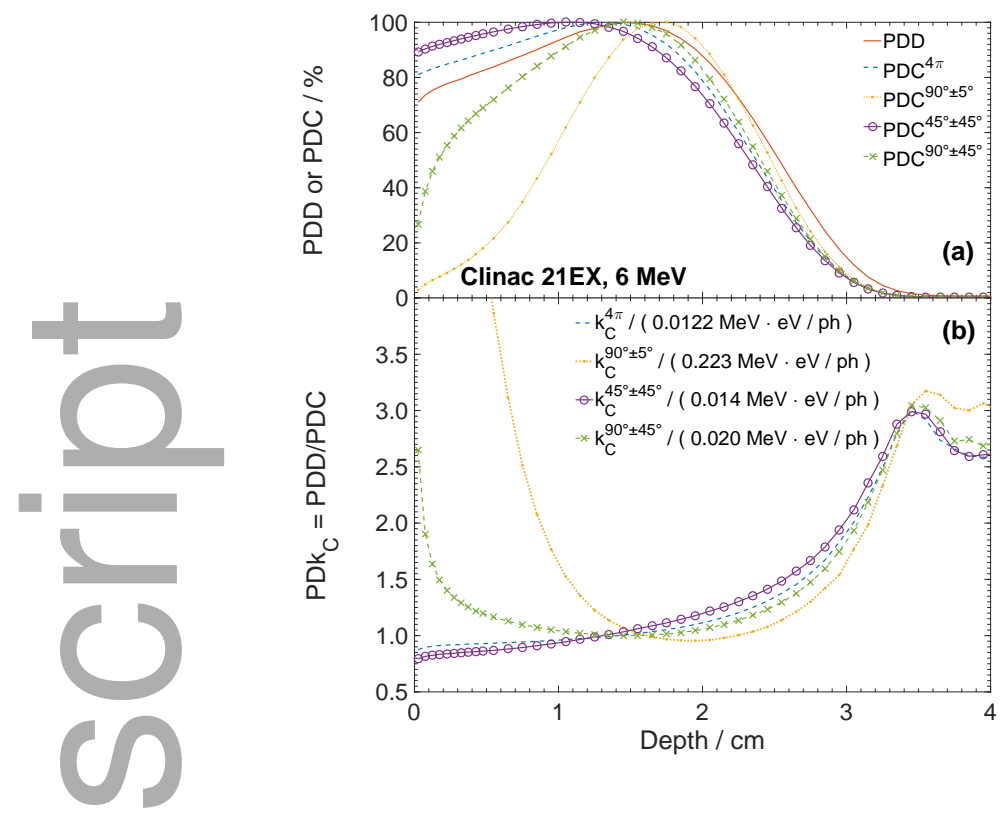

FIG. 4. Simulated (a) percent-depth dose, PDD, percent-depth Cherenkov emission (CE), $\mathrm{PDC}^{\theta \pm \delta \theta}$, and (b) their ratio, $\mathrm{PD} k_{\mathrm{C}}^{\theta \pm \delta \theta}$, equivalent to the CE-to-dose conversion, $k_{\mathrm{C}}^{\theta \pm \delta \theta}$, normalized by the dose-to-CE maximum ratio (given in the legend), in water, at polar angles $\theta \pm \delta \theta$ of $90^{\circ} \pm 90^{\circ}$ ( $4 \pi$ detection), $90^{\circ} \pm 5^{\circ}, 45^{\circ} \pm 45^{\circ}$, and $90^{\circ} \pm 45^{\circ}$ relative to the beam, for a Clinac 21EX $6 \mathrm{MeV}$ electron beam.
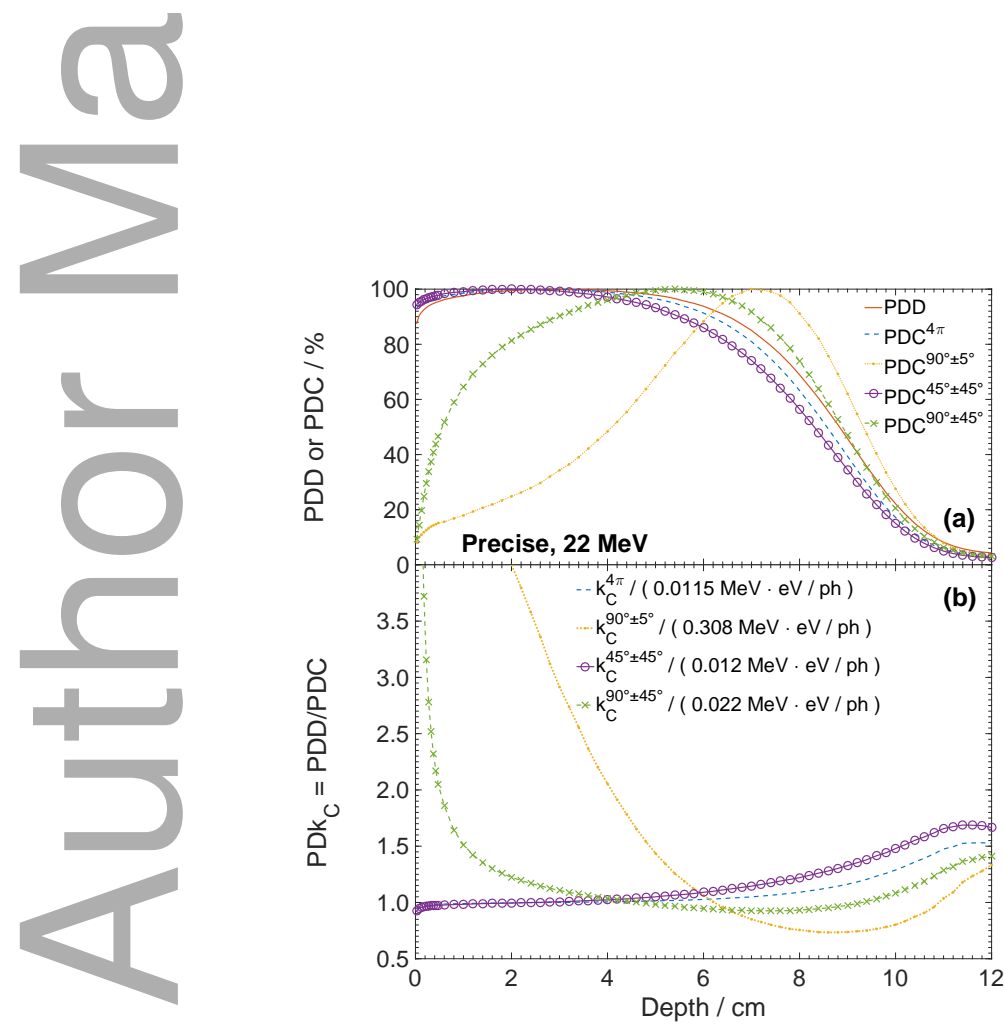

FIG. 5. Same as Figure 4 but for Precise $22 \mathrm{MeV}$ electrons. 

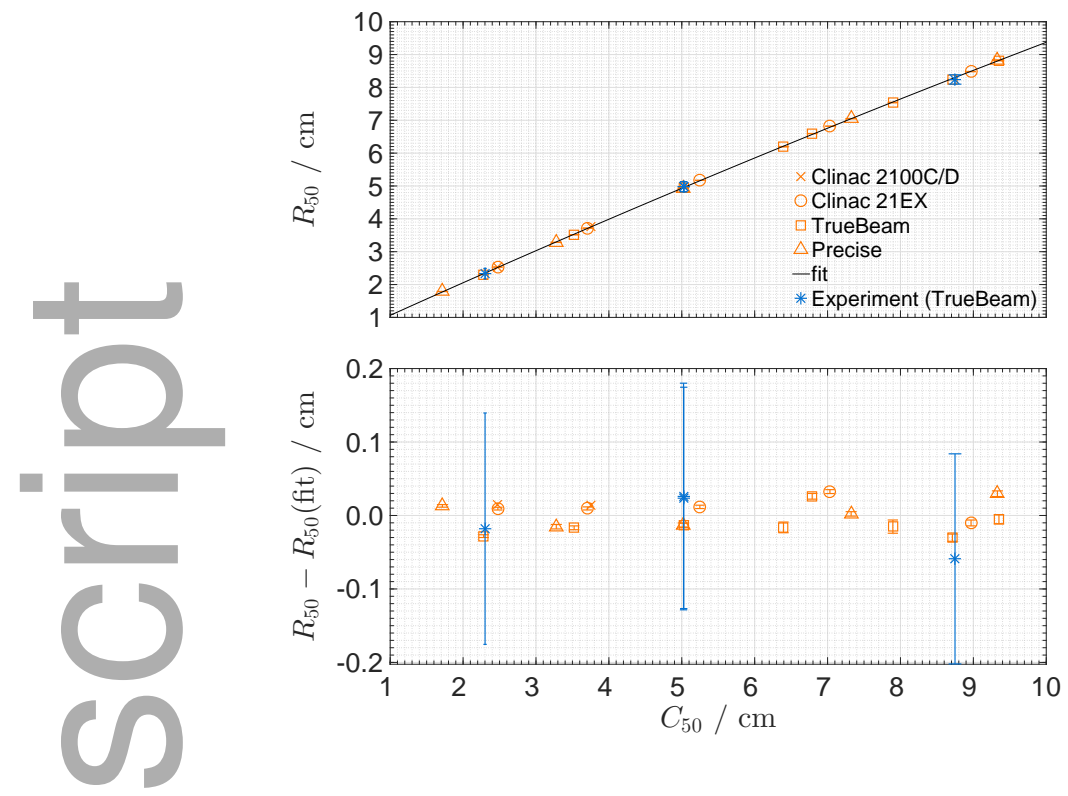

FIG. 6. Accuracy and experimental performance of fit to the Monte Carlo-calculated depth of $50 \%$ dose $R_{50}$ in terms of the depth of $50 \%$ Cherenkov $C_{50}$ for $90^{\circ} \pm 5^{\circ}$ detection as $R_{50}=-0.00860 C_{50}^{2}+1.018 C_{50}+0.053$ (in units of cm, Equation 2). The experimental error bars define an interval estimated to have $95 \%$ level of confidence, based on the t-distribution for 4 degrees of freedom ( 5 acquisitions), and are calculated solely from the experimental standard deviation of the mean.[6]
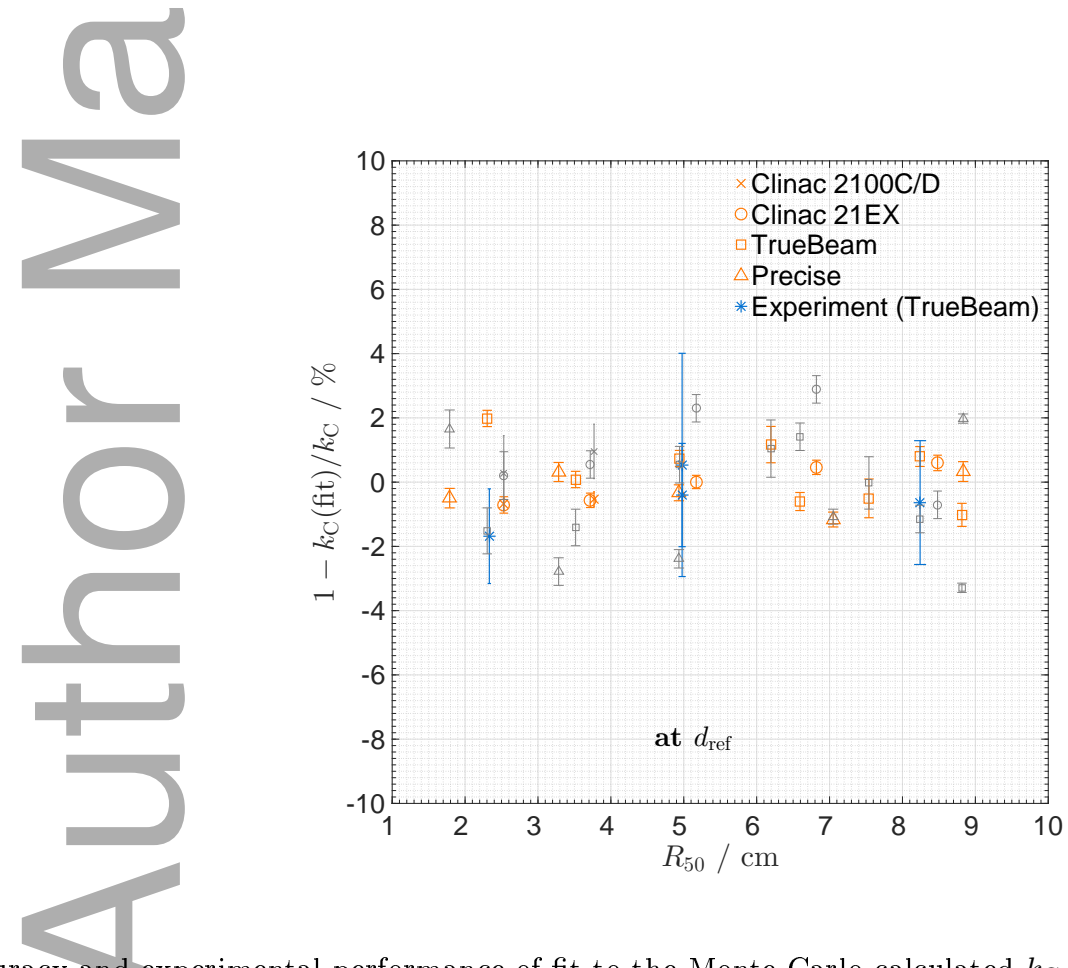

FIG. 7. Accuracy and experimental performance of fit to the Monte Carlo-calculated $k_{\mathrm{C}}$ (and PD $k_{\mathrm{C}}=\mathrm{PDD} / \mathrm{PDC}$ ) factors at the optimized reference depth for $90^{\circ} \pm 5^{\circ}$ detection, $d_{\text {ref }}=0.6 R_{50}-0.1 \mathrm{~cm}$, in terms of $R_{50}($ in units of $\mathrm{cm})$ as $k_{\mathrm{C}}\left(R_{50}, d_{\text {ref }}\right)=$ $0.2095\left(R_{50}\right)^{-0.3044}+0.03334\left(R_{50}\right)^{1.017}$ (in units of $\mathrm{MeV} \cdot \mathrm{eV} \cdot$ photon ${ }^{-1}$, Equation 3). The fit was optimized over absolute $k_{\mathrm{C}}$ factors at $d_{\text {ref }}$. The PDk fit accuracy (smaller gray markers) is shown here for reference and for comparison with experiments, which measured $\mathrm{PD} k_{\mathrm{C}}$. The experimental error bars are calculated as in Figure 6. 

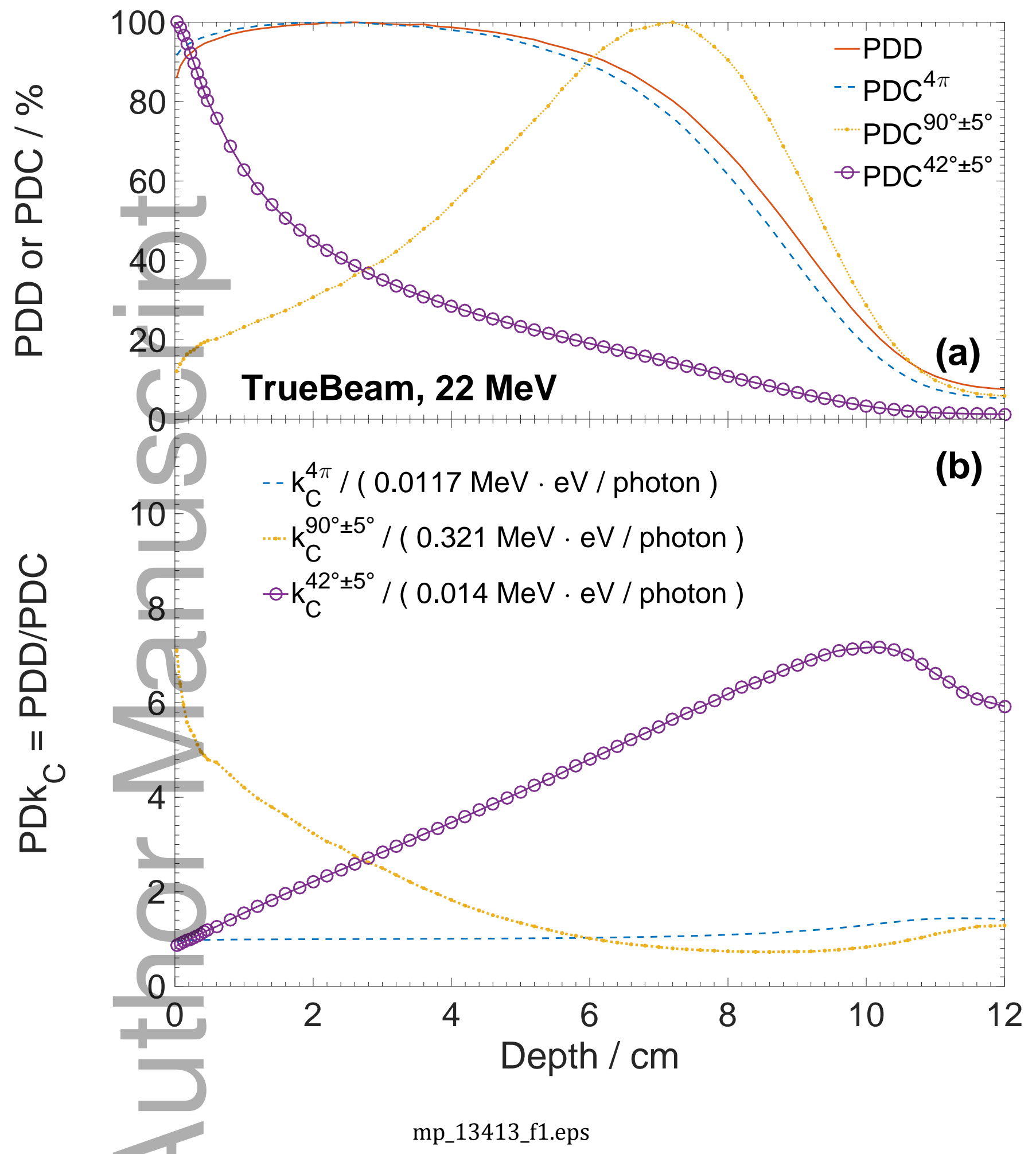

This article is protected by copyright. All rights reserved 


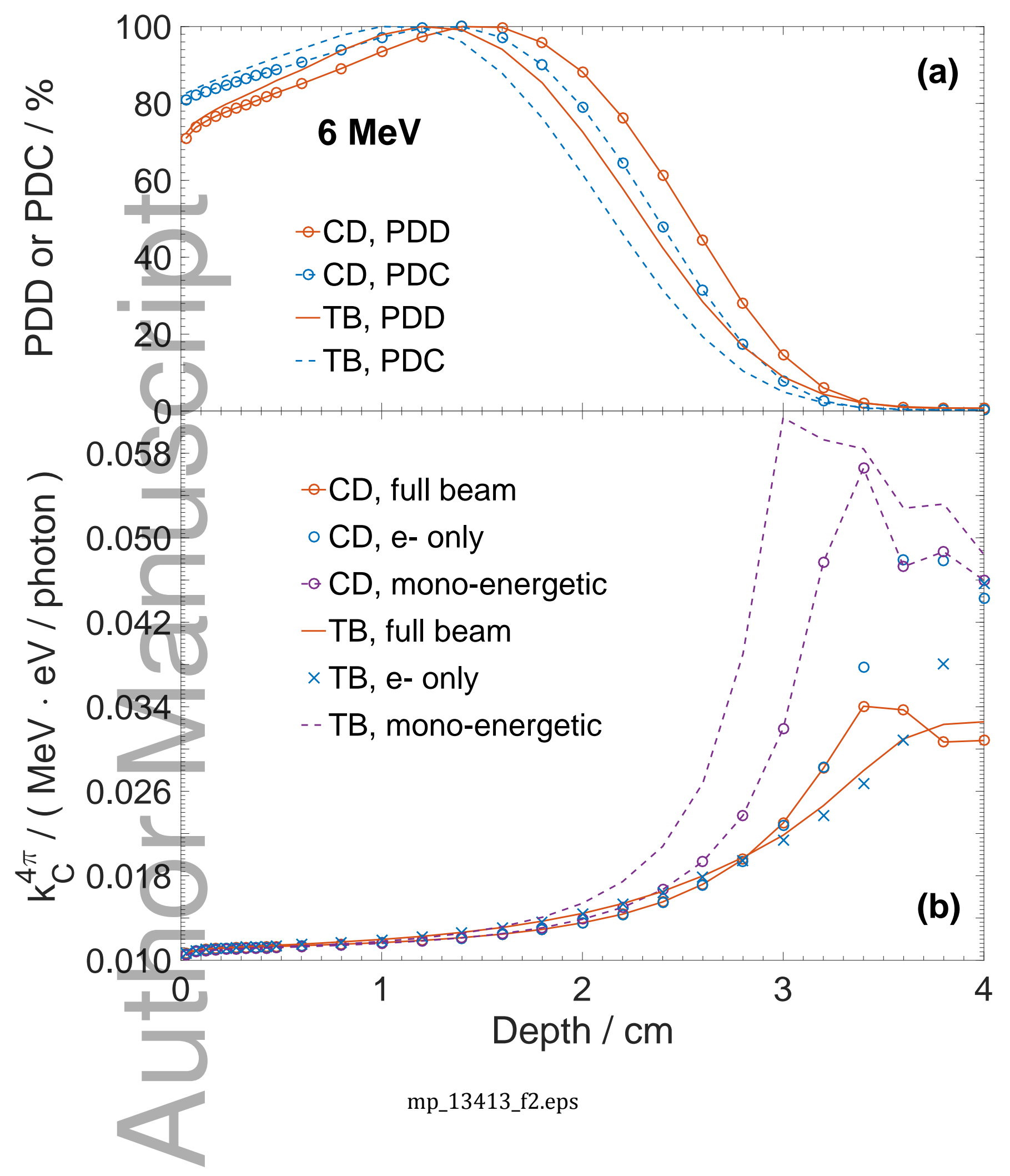

This article is protected by copyright. All rights reserved 


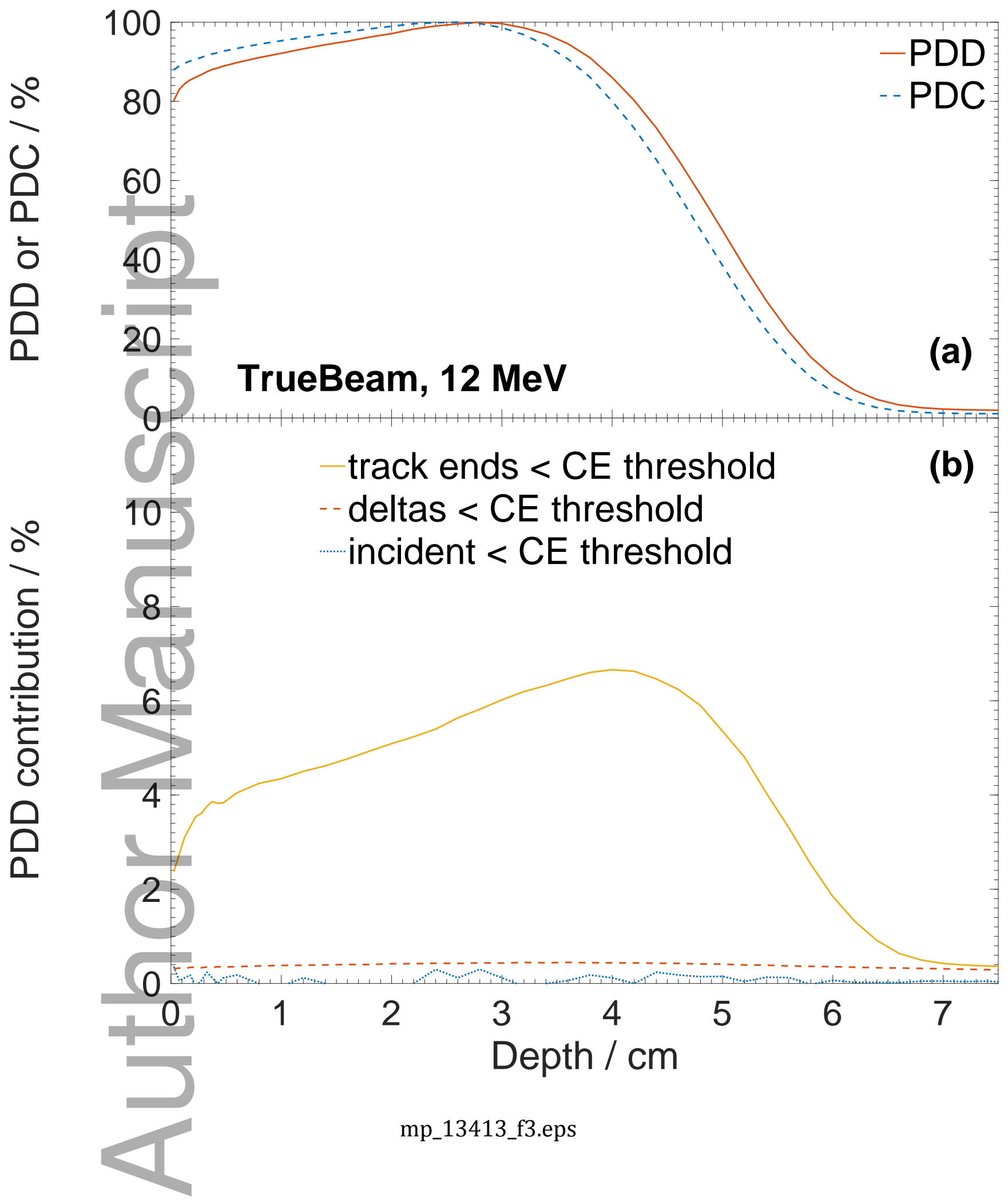

This article is protected by copyright. All rights reserved 


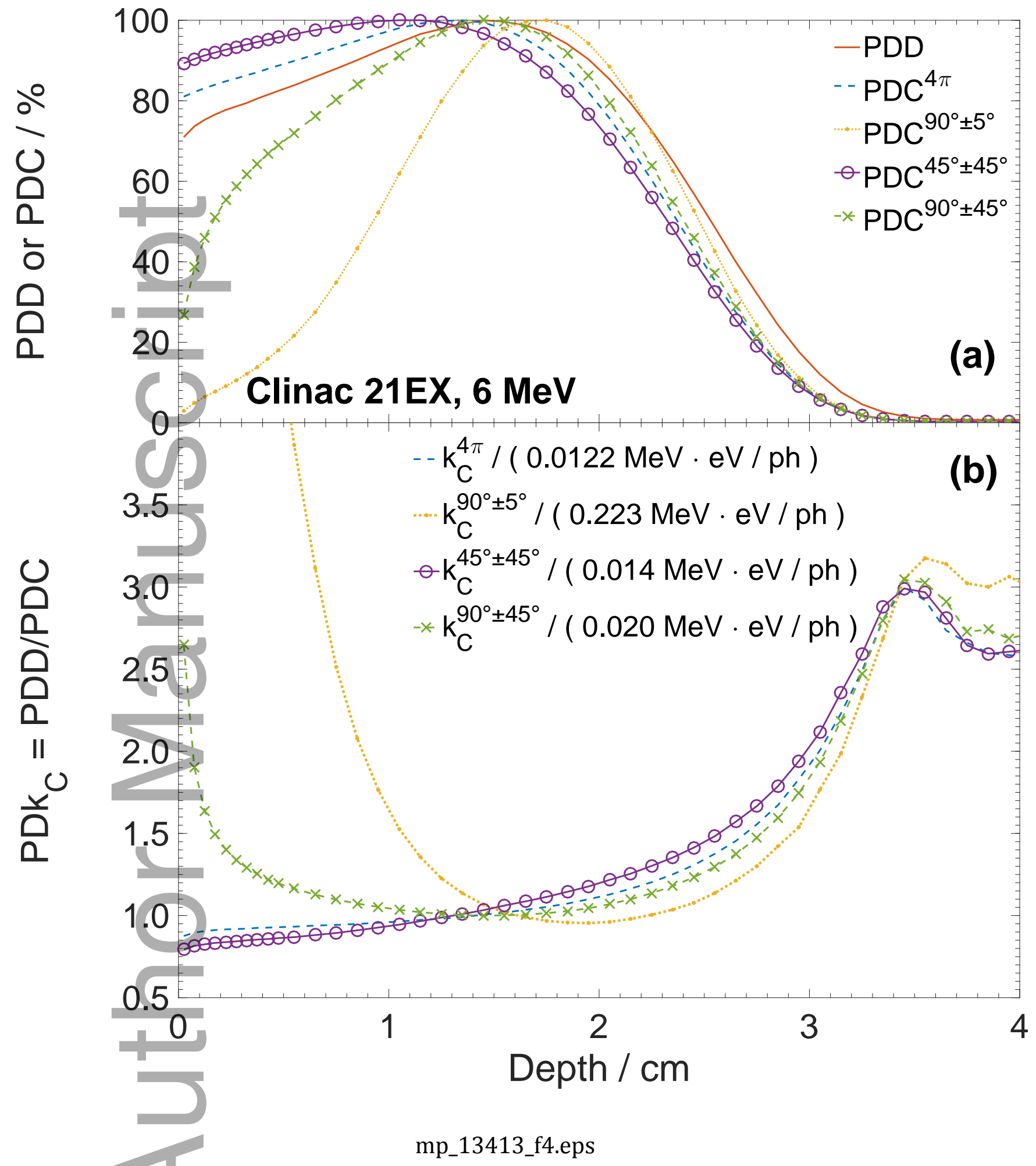

This article is protected by copyright. All rights reserved 


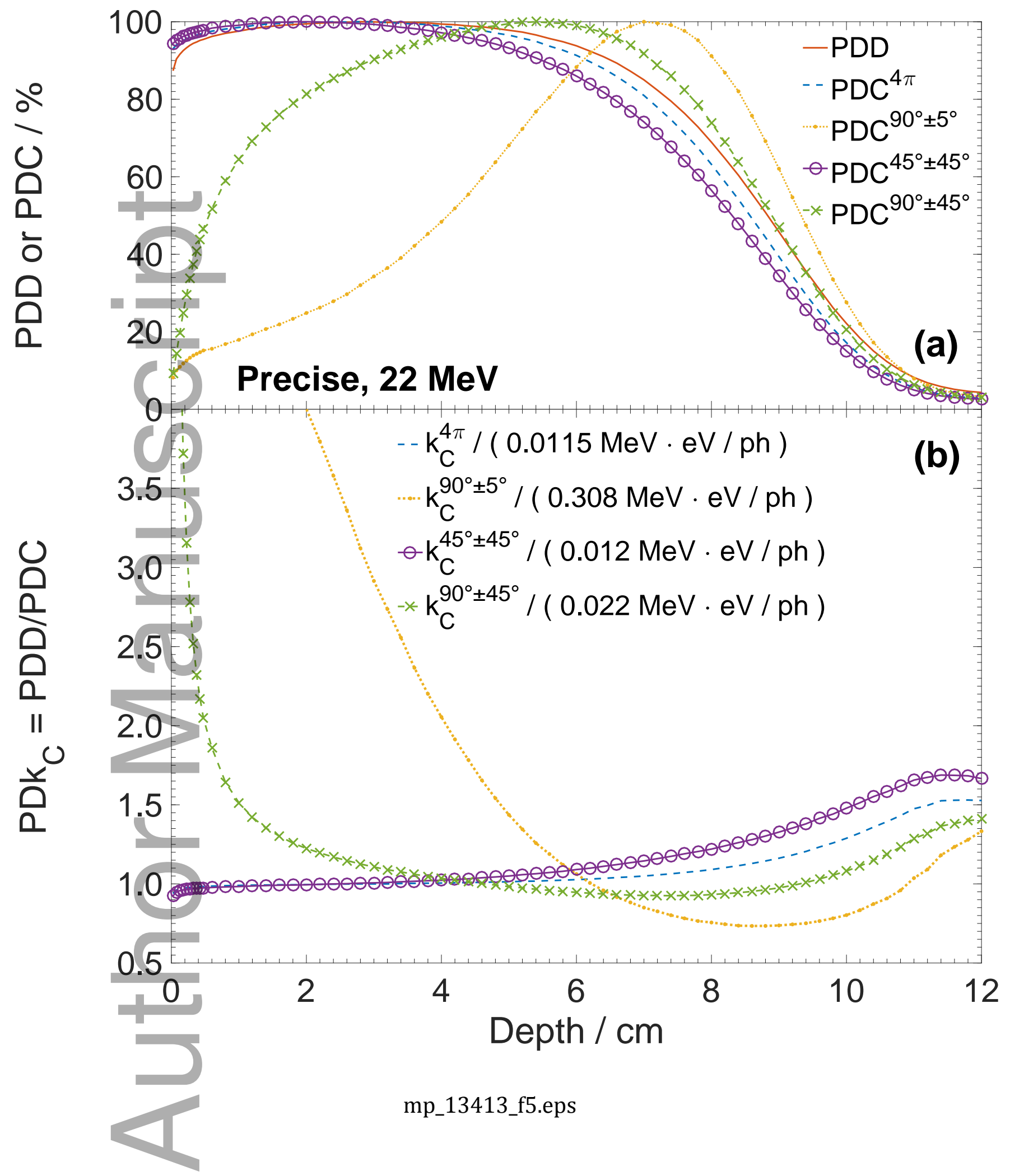

This article is protected by copyright. All rights reserved 

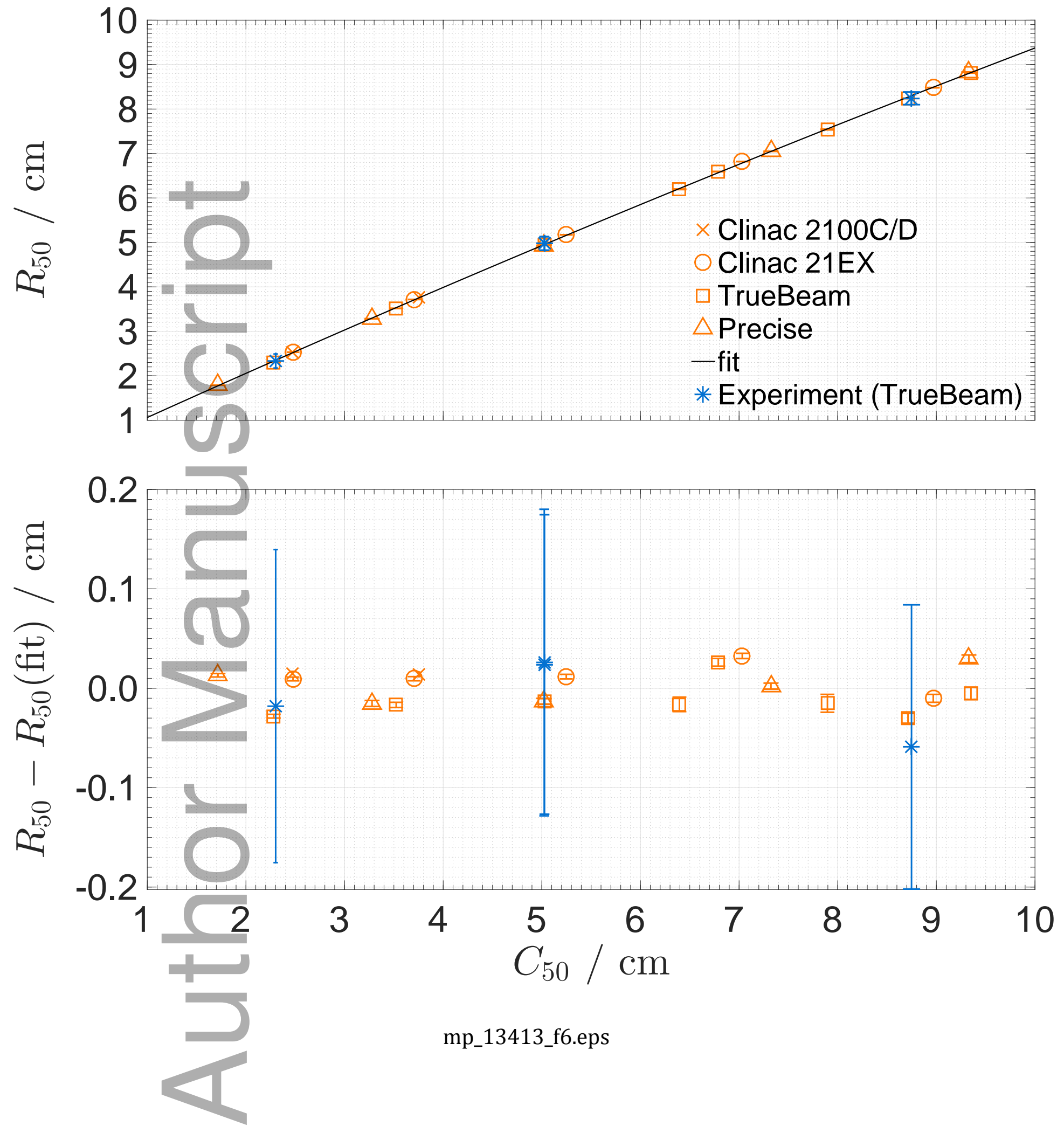

This article is protected by copyright. All rights reserved 


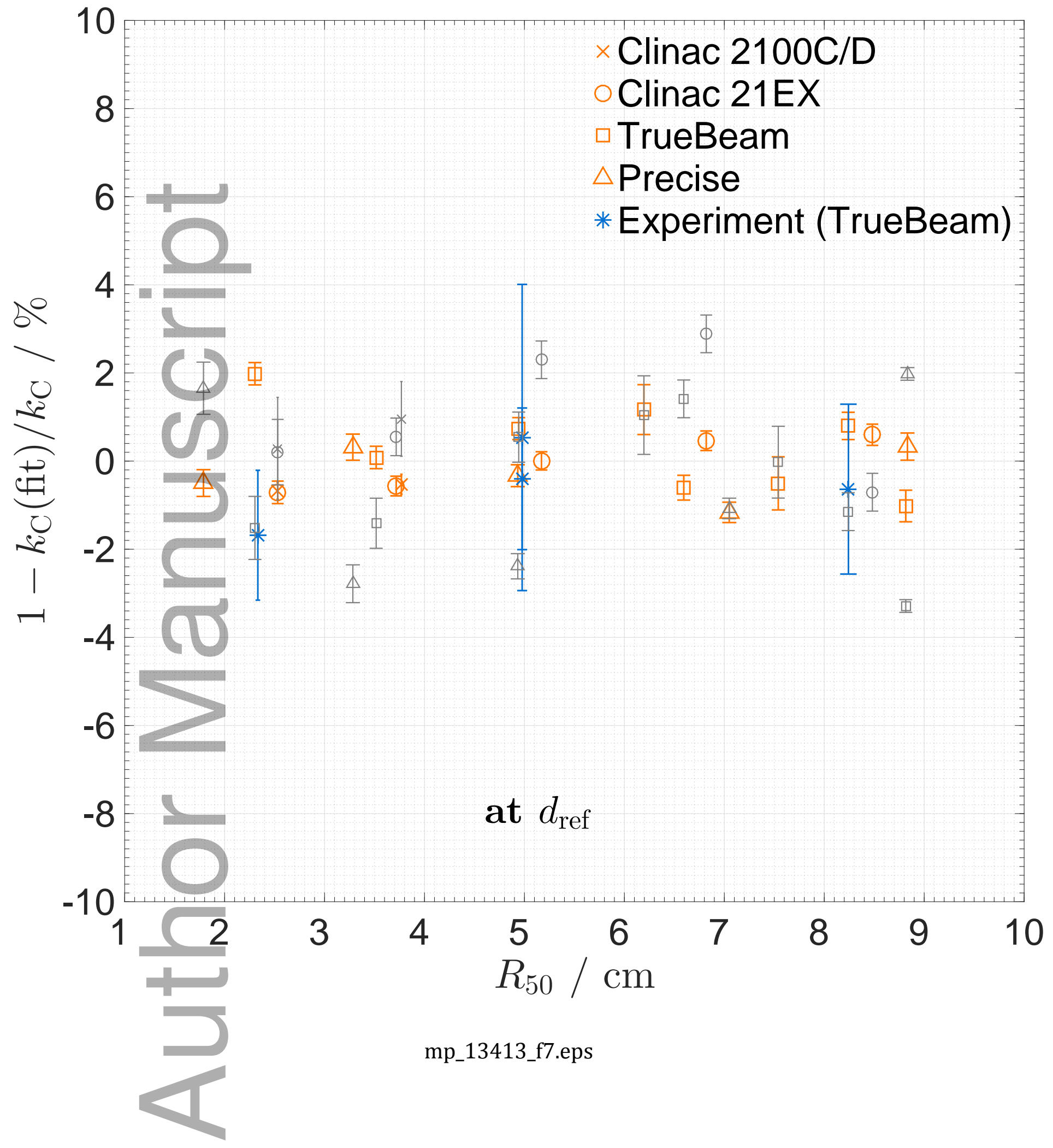

This article is protected by copyright. All rights reserved 